\title{
Characterization of Brain Dysfunction Induced by Bacterial Lipopeptides That Alter Neuronal Activity and Network in Rodent Brains
}

\author{
Kwang-Min Kim, ${ }^{1,2}$ Alsu I. Zamaleeva, ${ }^{1}$ Youn Woo Lee, ${ }^{3}$ ํ․ $\mathrm{M}$. Rafiuddin Ahmed, ${ }^{1}$ Eunkyung Kim, ${ }^{4}$ Hye-Ryeon Lee, ${ }^{2}$ \\ @Venkata Raveendra Pothineni, ${ }^{1}$ Juan Tao, ${ }^{1}$ Siyeon Rhee, ${ }^{6}$ Mithya Jayakumar, ${ }^{1}$ @Mohammed Inayathullah, ${ }^{1}$ \\ OSenthilkumar Sivanesan, ${ }^{1}$ Kristy Red-Horse, ${ }^{6}$ Theo D. Palmer, ${ }^{2}$ Jon Park, ${ }^{2}$ Daniel V. Madison, ${ }^{7}$ Ho-Young Lee, ${ }^{3,4 *}$ \\ and Jayakumar Rajadas ${ }^{1,5 *}$ \\ ${ }^{1}$ Biomaterials and Advanced Drug Delivery Laboratory, Stanford University School of Medicine, Palo Alto, California $94304,{ }^{2}$ Department of Neurosurgery, \\ Stanford University School of Medicine, Stanford, California 94305, 32Department of Nuclear Medicine, Seoul National University Bundang Hospital, \\ Seongnam, Korea, ${ }^{4}$ Department of Nuclear Medicine, Seoul National University, College of Medicine, Seoul, Korea, ${ }^{5}$ Department of Bioengineering and \\ Therapeutic Sciences, School of Pharmacy, University of California, San Francisco, California 94158, ${ }^{6}$ Department of Biology, and 7 Department of Molecular \\ and Cellular Physiology, Stanford University, Stanford, California 94305
}

The immunopathological states of the brain induced by bacterial lipoproteins have been well characterized by using biochemical and histological assays. However, these studies have limitations in determining functional states of damaged brains involving aberrant synaptic activity and network, which makes it difficult to diagnose brain disorders during bacterial infection. To address this, we investigated the effect of $\mathrm{Pam}_{3} \mathrm{CSK}_{4}$ (PAM), a synthetic bacterial lipopeptide, on synaptic dysfunction of female mice brains and cultured neurons in parallel. Our functional brain imaging using PET with $\left[{ }^{18} \mathrm{~F}\right]$ fluorodeoxyglucose and $\left[{ }^{18} \mathrm{~F}\right]$ flumazenil revealed that the brain dysfunction induced by PAM is closely aligned to disruption of neurotransmitter-related neuronal activity and functional correlation in the region of the limbic system rather than to decrease of metabolic activity of neurons in the injection area. This finding was verified by in vivo tissue experiments that analyzed synaptic and dendritic alterations in the regions where PET imaging showed abnormal neuronal activity and network. Recording of synaptic activity also revealed that PAM reorganized synaptic distribution and decreased synaptic plasticity in hippocampus. Further study using in vitro neuron cultures demonstrated that PAM decreased the number of presynapses and the frequency of miniature EPSCs, which suggests PAM disrupts neuronal function by damaging presynapses exclusively. We also showed that PAM caused aggregation of synapses around dendrites, which may have caused no significant change in expression level of synaptic proteins, whereas synaptic number and function were impaired by PAM. Our findings could provide a useful guide for diagnosis and treatment of brain disorders specific to bacterial infection.

Key words: bacterial lipoprotein; brain infection; PET; regional correlation; synaptic dysfunction

Significance Statement

It is challenging to diagnose brain disorders caused by bacterial infection because neural damage induced by bacterial products involves nonspecific neurological symptoms, which is rarely detected by laboratory tests with low spatiotemporal resolution. To better understand brain pathology, it is essential to detect functional abnormalities of brain over time. To this end, we investigated characteristic patterns of altered neuronal integrity and functional correlation between various regions in mice brains injected with bacterial lipopeptides using PET with a goal to apply new findings to diagnosis of brain disorder specific to bacterial infection. In addition, we analyzed altered synaptic density and function using both in vivo and in vitro experimental models to understand how bacterial lipopeptides impair brain function and network. 


\section{Introduction}

Bacterial infection in the central nervous system (CNS) causes amyloid $\beta$ aggregation (Boelen et al., 2007), loss of synapses (Wippel et al., 2013), demyelination (Rambukkana et al., 2002), and neuronal death (Saijo et al., 2009), which involves diverse clinical manifestations such as fever, seizure, and altered mental status (van de Beek et al., 2004; Hildenbrand et al., 2009). Even after removing the source of infection, patients suffer from sequelae and progression of neurodegenerative diseases (Compston and Coles, 2008; Dersch et al., 2015) because the bacterial surface components still remain in tissues when bacteria are degraded by antibiotic therapies (Evans and Pollack, 1993; Bockenstedt et al., 2012).

The effect of bacterial surface components (e.g., lipoproteins, lipopolysaccharides) on the immunopathological states of the brain has been extensively studied by correlating inflammatory activities of microglia and astrocytes with neural tissue injuries (Nau and Bruck, 2002; Glass et al., 2010). Briefly, bacterial surface components trigger microglia to produce inflammatory mediators (e.g., cytokines, chemokines) via the Toll-like receptor (TLR) family (Akira and Takeda, 2004; O’Neill et al., 2013) and induce migration of immune cells across the blood-brain barrier (Ek et al., 2001; Nau and Bruck, 2002), which eventually leads to neural injury including death of neurons and glial cells (i.e., astrocytes, oligodendrocytes) (Ramesh et al., 2003; Cunningham et al., 2005; Parthasarathy et al., 2013). However, this evidence is not sufficient to define brain disorders specific to bacterial infection and often correlated with vague neurological symptoms (e.g., fatigue, headache, insomnia), which prevents early diagnosis and treatment of fatal infections in the brain such as meningoencephalitis (Oschmann et al., 1998; Feder et al., 2007; Dersch et al., 2015). The poor correlation of bacterial infection-specific brain disorders with laboratory evidence and clinical manifestations could be caused by the paucity of information that substantiates dysfunctional states of the brain during bacterial infection (Reuber et al., 2005; Czerniawski and Guzowski, 2014). To address this, it is essential to probe neuron-specific functional abnormalities such as synaptic damage and neural network disruption in response to bacterial products. Toward this goal, we determined whether $\mathrm{Pam}_{3} \mathrm{CSK}_{4}$ (PAM), a synthetic bacterial lipopeptide, altered synaptic density and network in the brain using both in vivo and in vitro models.

PAM has been widely used to study the virulent effect of bacterial lipoproteins in bacterial infectious diseases (e.g., Lyme disease, syphilis) because it mimics the $\mathrm{N}$ termini of lipoproteins from spirochetal bacteria (e.g., Borrelia burgdorferi, Treponema pallidum) (Lien et al., 1999), which activates inflammatory pathways of microglia and glial cells via TLR2 (Aliprantis et al., 1999;

Received March 20, 2017; revised Aug. 24, 2018; accepted Sept. 15, 2018.

Author contributions: K.-M.K. wrote the first draft of the paper; K.-M.K. and J.P. edited the paper; K.-M.K., D.V.M., H.-Y.L., and J.R. designed research; K.-M.K., A.I.Z., Y.W.L., M.R.A., E.K., H.-R.L., V.R.P., J.T., S.R., M.J., M.I., S.S., K.R.-H., T.D.P., J.P., D.V.M., and H.-Y.L. performed research; K.-M.K., A.I.Z., Y.W.L., E.K., H.-R.L., V.R.P., S.R., M.I., S.S., K.R.-H., and D.V.M. analyzed data; K.-M.K. and D.V.M. wrote the paper.

This work was supported by the Bay Area Lyme Foundation; the L.K. Whittier Foundation; the Laurel STEM Fund; ICT and Future Planning and Korea Mouse Phenotyping Project, Ministry of Science (Korea) (2013M3A9D5072560); and the National Institute of Mental Health-National Institutes of Health (Grant MH11176802 to D.V.M.). We thank Byung Seok Moon and Byung Chul Lee at Seoul National University Bundang Hospital for preparing $\left[{ }^{88} \mathrm{~F}\right]$ flumazenil radiopharmaceuticals for $\mathrm{PET} / \mathrm{CT}$ image acquisition.

The authors declare no competing financial interests.

*Correspondence should be addressed to either of the following: Jayakumar Rajadas, Rm163, 1050 Arastradero, Palo Alto, CA 94304, E-mail: jayraja@stanford.edu; or Ho-Young Lee, 82 Gumi-ro 173, Jeongja 3dong, Bundang-gu, Seongnam-si, Gyeonggi-do, Korea, E-mail: md1004@snu.ac.kr.

https://doi.org/10.1523/JNEUROSCI.0825-17.2018

Copyright $\odot 2018$ the authors $\quad 0270-6474 / 18 / 3810673-20 \$ 15.00 / 0$
Nau and Bruck, 2002). To examine the effect of PAM on neuronal activity and network, we used $\left[{ }^{18} \mathrm{~F}\right]$ fluorodeoxyglucose (FDG) and $\left[{ }^{18} \mathrm{~F}\right]$ flumazenil (FMZ) PET imaging techniques. FDG PET shows glucose metabolism of neurons by measuring the level of $\left[{ }^{18} \mathrm{~F}\right]$ FDG uptake and FMZ PET shows concentrations of GABAergic receptors on neurons by measuring binding potentials $\left(\mathrm{BP}_{\mathrm{ND}}\right)$ of $\left[{ }^{18} \mathrm{~F}\right]$ FMZ to GABA-benzodiazepine receptors (Ryvlin et al., 1998). FDG PET reveals general activity of brain functions in the widespread region while FMZ PET provides more neuron-specific information such as neurotransmitterrelated neuronal activity and correlation between focal regions in the brain (Hammers et al., 2003; Vivash et al., 2013; Kujala et al., 2015). Therefore, measuring $\left[{ }^{18} \mathrm{~F}\right]$ FMZ $\mathrm{BP}_{\mathrm{ND}}$ is often used to determine neuronal integrity, which indicates the density of neurons showing active neurotransmitter binding in the brain (Shiga et al., 2006; Ivenshitz and Segal, 2010). This approach is reliable in determining neuron-specific activity during infection because FMZ binding is not affected by acute inflammation even when GABAa receptors are expressed on activated microglia (Parente et al., 2017).

To further investigate the results from PET imaging at tissue and cellular level, we also examined the effect of PAM on alteration of synapses and dendrites in both brain slices and cultured neurons. Using the results from in vivo and in vitro assays, we discuss below the characteristic dysfunctions of neurons and brains induced by bacterial lipopeptides.

\section{Materials and Methods}

Experiments. All experiments using animals were conducted according to the Administrative Panel on Laboratory Animal Care guidelines at Stanford University and to the permission of International Animal Care and Use Committee at Seoul National University Bundang Hospital.

Stereotactic injection of bacterial lipopeptides. Female mice (6-week-old C57BL/6) were weighed and anesthetized with intraperitoneal injection of ketamine $(100-200 \mu \mathrm{g} / \mathrm{g}) / x y l a z i n e ~(10-20 \mu \mathrm{g} / \mathrm{g})$. Only female mice were used for this study because lipoproteins from spirochetal bacteria are known to induce greater severity in male mice (Brown et al., 1999; Bockenstedt et al., 2012). The anesthetized mouse was placed on a heating pad and the eyes were kept moistened with ointment. The fur on the skull was shaved and the skin was cleaned with $70 \%$ ethanol. Then, the mouse was placed in the stereotaxic apparatus (Kopf Instruments). To fix the head position of the mouse in the apparatus, both ear canals were aligned onto the ear bars and the incisors of the mouse were inserted into the incisor adapter. The target (CA1 in the left hemisphere, coordinates of ML: -1.25 , AP: -1.95 , DV: -1.21 from the bregma) on the skull was drilled until the bone was thin enough for the penetration of micropipette. The tip of the micropipette was located on the target and lowered until it touched the exposed dura mater. Slow and steady pressure was applied to the syringe (Hamilton) to inject $1 \mu \mathrm{l}$ of molecules. Injection molecules included vehicle (endotoxin-free water), $150 \mathrm{ng} / \mathrm{ml}$ PAM (PAM150; InvivoGen), $500 \mathrm{ng} / \mathrm{ml}$ PAM (PAM500; InvivoGen), and $1000 \mathrm{ng} / \mathrm{ml}$ iturin A (Itu1000; Sigma-Aldrich) for PET imaging, immunohistochemistry, electrophysiology, TUNEL assay, and Western blot. After injection, the mouse was kept in a warm place until it fully recovered from anesthesia.

Image acquisition of $\left[{ }^{18} \mathrm{~F}\right] \mathrm{FDG}$ and $\left[{ }^{18} \mathrm{~F}\right] \mathrm{FMZ}$ PET/CT. To acquire $\left[{ }^{18} \mathrm{~F}\right]$-FDG PET/CT image, each mouse ( $n=7$ in each group) of four groups stereotactically injected with vehicle, PAM150, PAM500, and Itu 1000 was fasted at least $6 \mathrm{~h}$. $\left[{ }^{18} \mathrm{~F}\right] \mathrm{FDG}(500 \pm 23 \mu \mathrm{Ci})$ was intravenously administered through the tail vein. After administration of $\left[{ }^{18} \mathrm{~F}\right]$ FDG, mouse was placed in the dimmed lighted cage for $60 \mathrm{~min}$. Each mouse was maintained under anesthesia with isoflurane (2.5\% flow rate) for the duration of the scan. Animals were positioned prone in the standard mouse bed. Limbs were positioned lateral to the body to acquire uniform CT images. Whole brain CT images were acquired with a microPET/CT scanner (nanoPET/CT, Bioscan). For CT image acquisition, the 
$\mathrm{x}$-ray source was set to $200 \mu \mathrm{A}$ and $45 \mathrm{kVp}$ with $0.5 \mathrm{~mm}$. The CT images were reconstructed using cone beam reconstruction with a Shepp filter with the cutoff at the Nyquist frequency and a binning factor of 4 , resulting in an image matrix of $480 \times 480 \times 632$ and a voxel size of $125 \mu \mathrm{m}$. To acquire $\left[{ }^{18} \mathrm{~F}\right]$ FMZ PET, a mouse from each group was positioned in the bed and image was acquired in dynamic mode after injection of $\left[{ }^{18} \mathrm{~F}\right]$ FMZ $(514 \pm 23 \mu \mathrm{Ci})$ through the tail vein for $90 \mathrm{~min}$. To avoid interference from previous $\left[{ }^{18} \mathrm{~F}\right]$ that has an $\sim 2 \mathrm{~h}$ half-life, $\left[{ }^{18} \mathrm{~F}\right]$ FDG PET/CT scans and $\left[{ }^{18} \mathrm{~F}\right]$ FMZ PET/CT scans were performed with $12 \mathrm{~h}$ intervals for each group (i.e., vehicle, PAM150, PAM500, and Itu1000) to acquire images within the same day window. Mice that had been scanned at day 1 after injection were used again for FDG and FMZ scans at day 7 after injection.

Analysis of $\left[{ }^{18} \mathrm{~F}\right]$ FDG and $\left[{ }^{18} \mathrm{~F}\right]$ FMZ PET/CT. The FDG brain image of each mouse was spatially normalized to FDG PET template image of mouse brain using PMOD version 3.7 (PMOD Technologies). After registration to template image, a 3D brain mask image was applied to images and all values outside of the brain were set to zero. For the FMZ brain image analysis, we used a simplified reference tissue model to make parametric images of $\mathrm{BP}_{\mathrm{ND}}$ and the pons was used as a reference tissue (Gunn et al., 1997). Each parametric image was spatially normalized to an MRI template image of mouse brain. Images were analyzed using SnPM (Wellcome Trust Centre for Neuroimaging). Registered images were analyzed based on $t$ test. Comparison between test and vehicle groups was performed after nonparametric permutation. Uncorrected $p<0.005$ was used as the statistical threshold (Choi et al., 2014).

Functional correlation and brain network construction. To make brain networks, we used 53 nodes that were represented by the 53 volumes of interest (VOIs) (Dorr et al., 2007). We extracted intensity-normalized FDG uptake and $\mathrm{BP}_{\mathrm{ND}}$ of FMZ in the VOIs of each mouse. With FDG uptake and $\mathrm{BP}_{\mathrm{ND}}$ of FMZ, correlation coefficients were obtained. Pearson's correlation coefficients $(r)$ between each pair of the VOIs were calculated in an intersubject manner and a correlation matrix $(53 \times 53)$ was obtained from each group (Choi et al., 2014).

To evaluate statistical differences of interregional correlation between the groups, we performed permutation test on all possible connections between nodes. Interregional correlation matrix of vehicle, PAM, and Itu groups were transformed to $Z$ scores using Fisher transformation. Randomly reassigned labels were permuted 5000 times for each of 53 VOIs and interregional correlation matrices were calculated, followed by Fisher transformation. We obtained type I error by the comparison between the observed $Z$ score for each connection and the $Z$ score from the permuted data. To determine statistically different connections between vehicle and test groups, a threshold was set as $p<0.005$.

Immunohistochemistry (IHC). Mice were anesthetized and perfused with PBS until tissues were cleared of blood at day 1 and day 7 after injection ( $n=4$ per each group). Then, mice were perfused with $10 \mathrm{ml}$ of $4 \%$ PFA in PBS. The fixed brains were immersed in $30 \%$ sucrose in PBS for $48 \mathrm{~h}$ at $4^{\circ} \mathrm{C}$. The brains were embedded in Tissue-Tek Optimal Cutting Temperature (Sakura) and then coronally sectioned at $35 \mu \mathrm{m}$ thickness on a cryostat (Leica CM 1950). The collected mice brain sections showing hippocampal regions were free-floated in PBS and incubated in the blocking solution containing 5\% normal donkey serum (Jackson ImmunoResearch Laboratories) and 0.3\% Triton X-100 (SigmaAldrich) in PBS. Sections were then incubated with primary antibodies diluted in blocking solution overnight at $4^{\circ} \mathrm{C}$. The dilution ratio of primary antibodies was as follows: rabbit polyclonal anti-Homer1 (Synaptic Systems, 1:300), guinea pig polyclonal anti-VGluT1 (Synaptic Systems, 1:500), and chicken polyclonal anti-MAP2 (Abcam, 1:1000). After sections were washed with PBS $(10 \mathrm{~min} \times 2)$ and blocking solution (20 min $\times 1$ ), DyLight405 anti-guinea pig (Jackson ImmunoResearch Laboratories), Alexa Fluor 488 anti-chicken (Jackson ImmunoResearch Laboratories), and Alexa Fluor 594 anti-rabbit (Jackson ImmunoResearch Laboratories) were added to samples at a dilution of 1:800 for secondary antibody incubation at room temperature for $1 \mathrm{~h}$. The immunostained slides were mounted with ProLong Gold antifade reagent (Life Technologies) and cured for $24 \mathrm{~h}$. Fluorescent images of immunostained sections were acquired at $5 \times, 40 \times$, and $63 \times$ magnifications with a Zeiss LSM 880 confocal microscope.
Neuron cultures. For primary rat hippocampal neuron cultures $(n=3$ or 4 per each group), hippocampal tissues were dissected from Sprague Dawley embryonic day 18 rats (Charles River Laboratories). Dissected tissues were chemically dissociated in papain (Worthington) dissolved in hibernate medium (without calcium) (BrainBits) for $30 \mathrm{~min}$ at $37^{\circ} \mathrm{C}$ (Kim et al., 2013). The chemically dissociated tissues were triturated using a fire-polished Pasteur pipette up to 30-40 times. The mechanically dissociated tissues were centrifuged at 1200-1500 rpm for $1 \mathrm{~min}$. The pelleted cells were reconstituted with serum-free neurobasal medium (Invitrogen) containing B27 supplement (Invitrogen) and GlutaMAX (Invitrogen). A total of $8 \times 10^{4}$ cells were seeded on $12 \mathrm{~mm}$ PDL-coated coverslips (Neuvitro) for immunocytochemistry and $1 \times$ $10^{6}$ cells were seeded in the well of 6-well plate for Western blot. To suppress the growth of glial cells, $10 \mu \mathrm{M}$ arabinoside was added to the serum-free medium for the first $10 \mathrm{~d}$ and neurons were cultured for 17-19 d until being treated with $\operatorname{PAM}(50,150,500,2500 \mathrm{ng} / \mathrm{ml})$ and Itu $(100,1000 \mathrm{ng} / \mathrm{ml})$ for $18 \mathrm{~h}$. Treated neurons were fixed by $4 \%$ PFA for 30 min for immunocytochemistry.

Immunocytochemistry (ICC). The fixed cells were washed with PBS and then permeabilized in $0.25 \%$ Triton X-100 for $2 \mathrm{~min}$. Permeabilized cells were blocked with 5\% normal donkey serum at room temperature for 30 min and then incubated with primary antibodies diluted in blocking solution overnight at $4^{\circ} \mathrm{C}$. The dilution ratio of primary antibodies was as follows: rabbit polyclonal anti-Homer1 (Synaptic Systems, 1:500), guinea pig polyclonal anti-VGluT1 (Synaptic Systems, 1:5000), and chicken polyclonal anti-MAP2 (Abcam, 1:10000). Incubated samples were washed with $1 \times$ PBS $(5 \mathrm{~min} \times 2)$ and blocking solution $(30 \mathrm{~min} \times$ 1). Then, Alexa Fluor 488 anti-chicken (Jackson ImmunoResearch Laboratories), Alexa Fluor 594 anti-rabbit (Jackson ImmunoResearch Laboratories), and DyLight405 anti-guinea pig (Jackson ImmunoResearch Laboratories) were added to samples at a dilution of 1:800 for secondary antibody incubation at room temperature for $1 \mathrm{~h}$. The immunostained slides were mounted with ProLong Gold antifade reagent (Thermo Fisher Scientific) and cured for $24 \mathrm{~h}$.

Analysis of synaptic and dendritic alterations in tissue sections. All sections to be analyzed were obtained from four independent injections for each condition. All images were captured at $63 \times$ magnification with a Zeiss LSM880 confocal microscope under identical condition (e.g., exposure time, gain intensity) for three fluorescent channels ( $\mathrm{WL}=405$, 488, and 594).

To measure synaptic density within the selected region from the section, we calculated the integrated density of fluorescent intensity in the region of interest measured by ImageJ software (available at http:// imagej.nih.gov/ij/). The value of integrated density of fluorescent intensity was obtained with the following equation: Integrated density of fluorescent intensity in the region of interest = integrated density (VGluT1 or Homer1) of selected region - (area of selected region $\times$ mean fluorescence value from the background [the lowest (darkest) signal in the tissue section)].

To measure the density of dendrites within the region of interest, we performed the same calculations as the analysis of synaptic density. To measure the coverage of dendritic area density in the region of interest, we used the "Area \% measurement" function provided by ImageJ.

Analysis of synaptic density in tissue sections and neuron cultures. Fluorescent images of immunostained samples were acquired at $63 \times$ magnification with a Zeiss LSM880 confocal microscope. For imaging synapses in brain slices, an additional digital zoom (X1.2-1.8) and the Airyscan function in the microscope were applied to provide a better resolution on synaptic structures. The particle analyzer in ImageJ (1.46r) (available at http://imagej.nih.gov/ij/) was used for counting the synaptic proteins in the selected dendrite. The average value of synaptic puncta was obtained by the total number of synaptic puncta divided by the length of dendrite (MAP2) selected.

Analysis of dendrite morphology. The dendrite morphology of cultured neurons treated with vehicle, PAM150, PAM500, PAM2500, and Itu1000 were analyzed with respect to the following: (1) the number of primary dendrites, (2) the extension range of apical dendrite, (3) the number of branch nodes per apical/basal dendrite, and (4) the number of dendrite intersections. For the analysis of dendrite intersections, dendrites of neu- 
A $\left[{ }^{18} \mathrm{~F}\right]$ FDG PET

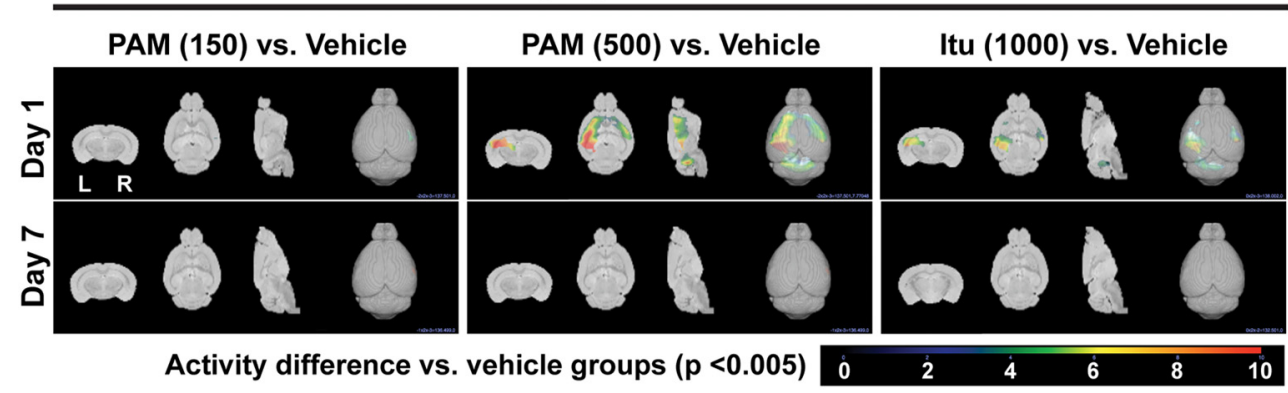

B

$\left[{ }^{18} \mathrm{~F}\right]$ FMZ PET

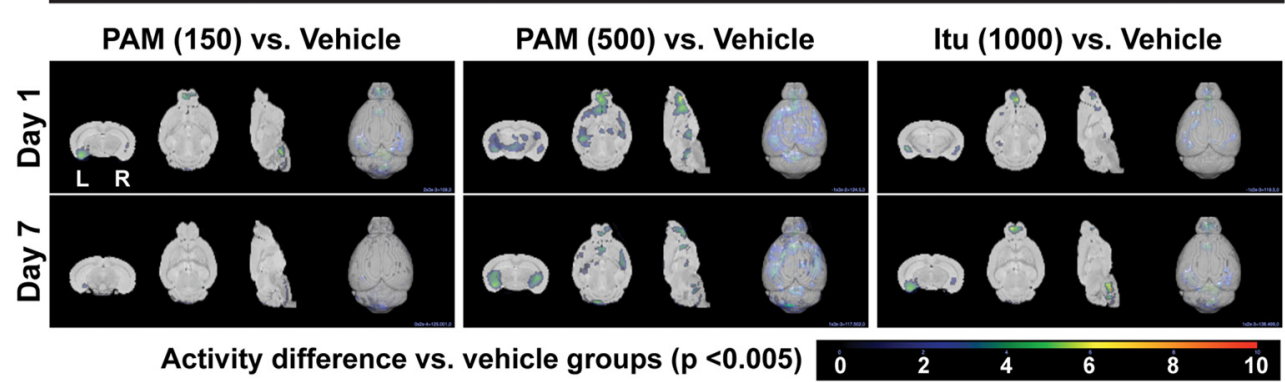

Figure 1. Metabolic activity and neuronal integrity map of mice brains for $7 \mathrm{~d}$ following stereotactic injection of PAM and Itu into the leftCA1. A, FDG PET images showing altered metabolic activity of neurons for $7 \mathrm{~d}(p<0.005$ vs vehicle). Both PAM and Itu altered metabolic activity compared with vehicle groups at day 1. PAM500 induced severe abnormalities in hippocampal regions (i.e., injection site). However, all groups showing abnormal metabolic activity recovered to the level of vehicle group in 1 week. $B$, FMZ PET images showing altered neuronal integrity for $7 \mathrm{~d}$ ( $p<0.005$ vs vehicle). Both PAM and Itu altered neuronal integrity at day 1. PAM500 resulted in abnormal neuronal integrity globally in the brain, whereas PAM150 and Itu1000 showed local effects around EC. At day 7, abnormal neuronal integrity still remained in hippocampus and EC by PAM 500 and in EC by Itu 1000 . Heat maps represent Z-score between vehicle and test groups ( $p<0.005$ vs vehicle, $n=7$ mice per each group).

rons stained with anti-MAP2 were manually traced on the template circles ranging from 20 to $200 \mu \mathrm{m}$ with $20 \mu \mathrm{m}$ per step from the center of the soma.

Electrophysiology of hippocampal slices. Fresh hippocampal slices were prepared before every experiment. The left (treated side) hippocampus was dissected from the brain $7 \mathrm{~d}$ after injection of vehicle, PAM (500 $\mathrm{ng} / \mathrm{ml})$ or Itu $(1000 \mathrm{ng} / \mathrm{ml})$ and placed in ice-cold artificial CSF (ACSF). The composition of the ACSF was as follows (in mM): $119 \mathrm{NaCl}, 2.5 \mathrm{KCl}$, $1.3 \mathrm{MgSO}_{4} 2.5 \mathrm{CaCl} 2,1 \mathrm{Na} 2 \mathrm{HPO}_{4}, 26.2 \mathrm{NaHCO}$, and 11 glucose pre-gassed with $95 \% \mathrm{O}_{2}$ and $5 \% \mathrm{CO}_{2}$. The hippocampus was then placed on the stage of a manual tissue slicer (Stoelting) and cut into $500 \mu \mathrm{m}$ slices, which were stored on Whatman \#2 filter paper wet with ACSF inside a chamber charged with moist $95 \% \mathrm{O}_{2} / 5 \% \mathrm{CO}_{2}$ ("carbogen"). After $2 \mathrm{~h}$ of recovery, a slice was placed into a submerged Zbic/Haas-type recording chamber (Harvard Apparatus), where it was continuously superfused with carbogen-saturated ACSF warmed to $30^{\circ} \mathrm{C}$. A bipolar concentric stimulating electrode (Frederick Haer) was placed into the stratum radiatum near the CA2/CA1 border. A fine glass recording pipette filled with saturated $\mathrm{NaCl}(\sim 1 \mathrm{M} \Omega$ resistance $)$ was also placed in the stratum radiatum in area CA1, $\sim 2 / 3$ of the distance from CA 2 to subiculum. EPSP field potentials were evoked by single $100 \mu$ s shocks through the stimulating electrode, one every $15 \mathrm{~s}$. Input/output (I/O) curves were obtained by progressively increasing the stimulus strength through a series of proscribed steps between $0.01 \mathrm{~mA}$ to $1 \mathrm{~mA}$ (constant current). Five measurements at each stimulus strength were taken and averaged in each. Illustrated I/O curves are averages of these averages across all slices. LTP was induced in a subset of slices by applying a theta-burst stimulation (bursts of 4 pulses at $100 \mathrm{hz}, 5$ bursts per second for 16 bursts).

The rising initial slope of the field EPSP (fEPSP) was measured as an index of synaptic strength. In most, but not all, recordings, a clear presynaptic fiber volley was also measured (amplitude in millivolts). In experiments in which we illustrate a fiber volley versus fEPSP I/O curve, we only used recordings in which both could be measured (fiber volleys are sometimes too small or too obscured by the EPSP to measure; in those cases, they are not included). In those rare cases where there was some ambiguity as whether a particular field potential rising phase was fiber volley or EPSP, we applied a pair-pulse protocol to differentiate between them (EPSPs facilitate, fiber volleys do not). The statistical significance of difference between $\mathrm{I} / \mathrm{O}$ curves was tested by converting them to cumulative curves and applying the Kolmogorov-Smirnov (K-S) test. For LTP, ANOVA for repeated measures was used, with significance level in both cases at $p<0.05$.

Whole-cell patch-clamp recording in cultured neurons. For whole-cell patch-clamp recording in cultured hippocampal neurons (17-19 d in vitro, DIV), ACSF containing the following (in mM): $125 \mathrm{NaCl}, 2.5 \mathrm{KCl}$, $1.25 \mathrm{NaH}_{2} \mathrm{PO}_{4}, 25 \mathrm{NaHCO}_{3}, 15$ glucose, $2 \mathrm{CaCl}_{2}$, and $1 \mathrm{MgCl}_{2}$ was bathapplied during recording session and oxygenated with $95 \% \mathrm{O}_{2}$ and $5 \%$ $\mathrm{CO}_{2}(\sim 300-305$ mOsm, pH 7.4). After PAM incubation, cultured hippocampal neurons were transferred to a submerged recording chamber perfused with ACSF at a rate of $\sim 2-3 \mathrm{ml} / \mathrm{min}$ at room temperature. Neurons were identified by IR-DIC (BX51, Olympus). Whole-cell voltage-clamp recordings were made with borosilicate glass pipettes $(\sim 3-4 \mathrm{M} \Omega$ ) filled with an internal solution containing the following (in mM): $126 \mathrm{CsMeSO}_{3}, 10$ HEPES, 1 EGTA, 2 QX-314 chloride, $0.1 \mathrm{CaCl}_{2}, 4$ MgATP, $0.3 \mathrm{Na}_{3} \mathrm{GTP}$, and $8 \mathrm{Na}_{2}$-phosphocreatine, $\sim 280-290 \mathrm{mOsm}$, $\mathrm{pH} 7.3$ with $\mathrm{CsOH}$. To measure miniature EPSCs (mEPSCs), neurons were held at $-65 \mathrm{mV}$ and TTX $(1 \mu \mathrm{M})$ and picrotoxin $(100 \mu \mathrm{M})$ were always present to block Nav and $\mathrm{GABA}_{\mathrm{A}}$ receptors, respectively. Access resistance was $\sim 10-20 \mathrm{M} \Omega$ and only cells with a change in access resistance $<20 \%$ were included in the analysis. Whole-cell patch recordings were performed using Multiclamp 700B (Molecular Devices), monitored (WinWCP, Strathclyde Electrophysiology Software), and analyzed offline using Clampfit version 10.0 (Molecular Devices) and Mini analysis program (Synaptosoft). Signals were filtered at $2 \mathrm{kHz}$ and digitized at 10 kHz (NI PCIe-6259, National Instruments). Drugs (TTX, picrotoxin) were purchased from Tocris Bioscience. 
Table 1. FMZ BP

\begin{tabular}{|c|c|c|c|c|c|c|c|c|c|}
\hline & & Vehicle & & PAM150 & & PAM500 & & ITU1000 & \\
\hline & Region & Day 1 & Day 7 & Day 1 & Day 7 & Day 1 & Day 7 & Day 1 & Day 7 \\
\hline 1 & L. amygdala & $0.67 \pm 0.08$ & $0.69 \pm 0.09$ & $0.64 \pm 0.04$ & $0.55 \pm 0.08$ & $0.55 \pm 0.09$ & $0.57 \pm 0.01$ & $0.79 \pm 0.07$ & $0.72 \pm 0.06$ \\
\hline 2 & L. frontal lobe & $1.07 \pm 0.10$ & $1.04 \pm 0.12$ & $0.91 \pm 0.02$ & $0.94 \pm 0.19$ & $0.89 \pm 0.30$ & $0.90 \pm 0.22$ & $1.18 \pm 0.08$ & $1.22 \pm 0.15$ \\
\hline 3 & L. optic nerve & $0.35 \pm 0.06$ & $0.36 \pm 0.08$ & $0.39 \pm 0.03$ & $0.28 \pm 0.06$ & $0.24 \pm 0.11$ & $0.29 \pm 0.11$ & $0.42 \pm 0.06$ & $0.41 \pm 0.06$ \\
\hline 4 & L. ant. comm. & $1.24 \pm 0.08$ & $1.30 \pm 0.10$ & $1.24 \pm 0.10$ & $1.18 \pm 0.17$ & $1.08 \pm 0.32$ & $1.11 \pm 0.21$ & $1.39 \pm 0.10$ & $1.47 \pm 0.13$ \\
\hline 5 & L. cerebellum & $0.40 \pm 0.06$ & $0.43 \pm 0.07$ & $0.40 \pm 0.07$ & $0.39 \pm 0.13$ & $0.36 \pm 0.15$ & $0.46 \pm 0.13$ & $0.47 \pm 0.07$ & $0.54 \pm 0.08$ \\
\hline 6 & L. corpus callosum & $1.84 \pm 0.16$ & $1.88 \pm 0.27$ & $1.77 \pm 0.03$ & $1.73 \pm 0.34$ & $1.65 \pm 0.43$ & $1.73 \pm 0.25$ & $2.11 \pm 0.24$ & $2.08 \pm 0.27$ \\
\hline 7 & L. fasci. retroflexus & $0.52 \pm 0.06$ & $0.49 \pm 0.06$ & $0.48 \pm 0.07$ & $0.52 \pm 0.15$ & $0.53 \pm 0.15$ & $0.48 \pm 0.17$ & $0.47 \pm 0.07$ & $0.50 \pm 0.07$ \\
\hline 8 & L. fonix & $1.14 \pm 0.11$ & $1.18 \pm 0.11$ & $1.09 \pm 0.23$ & $0.98 \pm 0.43$ & $0.95 \pm 0.43$ & $1.29 \pm 0.23$ & $1.31 \pm 0.24$ & $1.21 \pm 0.24$ \\
\hline 9 & L. globus pallidus & $1.01 \pm 0.06$ & $1.01 \pm 0.10$ & $1.03 \pm 0.03$ & $0.98 \pm 0.18$ & $0.91 \pm 0.24$ & $0.96 \pm 0.07$ & $1.15 \pm 0.15$ & $1.19 \pm 0.12$ \\
\hline 10 & L. hippocampus & $1.74 \pm 0.09$ & $1.81 \pm 0.17$ & $1.70 \pm 0.04$ & $1.66 \pm 0.22$ & $1.56 \pm 0.33$ & $1.61 \pm 0.17$ & $2.00 \pm 0.18$ & $1.92 \pm 0.17$ \\
\hline 11 & L. hypothal. & $0.59 \pm 0.06$ & $0.60 \pm 0.10$ & $0.56 \pm 0.01$ & $0.53 \pm 0.07$ & $0.47 \pm 0.12$ & $0.52 \pm 0.06$ & $0.66 \pm 0.08$ & $0.60 \pm 0.05$ \\
\hline 12 & L. int. capsule & $0.89 \pm 0.06$ & $0.94 \pm 0.09$ & $0.90 \pm 0.04$ & $0.90 \pm 0.12$ & $0.81 \pm 0.19$ & $0.90 \pm 0.07$ & $1.06 \pm 0.13$ & $1.06 \pm 0.10$ \\
\hline 13 & L. mammilothal. tr. & $0.93 \pm 0.11$ & $0.94 \pm 0.13$ & $0.85 \pm 0.01$ & $0.89 \pm 0.06$ & $0.78 \pm 0.17$ & $0.84 \pm 0.18$ & $0.98 \pm 0.09$ & $0.92 \pm 0.11$ \\
\hline 14 & L. medulla & $0.00 .2 \pm 0.01$ & $0.03 \pm 0.01$ & $0.02 \pm 0.01$ & $0.02 \pm 0.01$ & $0.03 \pm 0.01$ & $0.02 \pm 0.01$ & $0.03 \pm 0.01$ & $0.02 \pm 0.01$ \\
\hline 15 & L. midbrain & $1.60 \pm 0.09$ & $1.64 \pm 0.18$ & $1.60 \pm 0.16$ & $1.53 \pm 0.26$ & $1.44 \pm 0.30$ & $1.58 \pm 0.30$ & $1.78 \pm 0.17$ & $1.76 \pm 0.20$ \\
\hline 16 & L. occ. cortex & $1.04 \pm 0.14$ & $1.06 \pm 0.15$ & $1.06 \pm 0.05$ & $0.96 \pm 0.32$ & $0.90 \pm 0.28$ & $1.18 \pm 0.26$ & $1.16 \pm 0.12$ & $1.23 \pm 0.10$ \\
\hline 17 & L. olf. bulb & $1.02 \pm 0.11$ & $1.05 \pm 0.07$ & $0.92 \pm 0.03$ & $0.92 \pm 0.16$ & $0.85 \pm 0.29$ & $0.82 \pm 0.25$ & $1.10 \pm 0.13$ & $1.23 \pm 0.19$ \\
\hline 18 & L.P-T cortex & $1.05 \pm 0.10$ & $1.06 \pm 017$ & $0.94 \pm 0.19$ & $0.91 \pm 0.22$ & $0.83 \pm 0.25$ & $0.97 \pm 0.12$ & $1.19 \pm 0.08$ & $1.12 \pm 0.10$ \\
\hline 19 & L.pons & $0.19 \pm 0.01$ & $0.19 \pm 0.01$ & $0.18 \pm 0.00$ & $0.18 \pm 0.01$ & $0.17 \pm 0.01$ & $0.19 \pm 0.01$ & $0.20 \pm 0.01$ & $0.21 \pm 0.01$ \\
\hline 20 & L. str. med. thal. & $0.62 \pm 0.13$ & $0.76 \pm 0.10$ & $0.71 \pm 0.11$ & $0.74 \pm 0.12$ & $0.62 \pm 0.17$ & $0.74 \pm 0.05$ & $0.86 \pm 0.15$ & $0.84 \pm 0.09$ \\
\hline 21 & L. striatum & $1.39 \pm 0.07$ & $1.39 \pm 0.16$ & $1.44 \pm 0.05$ & $1.31 \pm 0.22$ & $1.30 \pm 0.28$ & $1.36 \pm 0.12$ & $1.59 \pm 0.17$ & $1.67 \pm 0.19$ \\
\hline 22 & L. sub. region (P-T) & $0.86 \pm 0.06$ & $0.88 \pm 0.11$ & $0.85 \pm 0.05$ & $0.77 \pm 0.11$ & $0.70 \pm 0.17$ & $0.76 \pm 0.06$ & $0.97 \pm 0.10$ & $1.03 \pm 0.10$ \\
\hline 23 & L. sub. region frontal & $0.84 \pm 0.06$ & $0.86 \pm 0.10$ & $0.81 \pm 0.05$ & $0.79 \pm 0.12$ & $0.71 \pm 0.19$ & $0.79 \pm 0.10$ & $0.97 \pm 0.12$ & $0.95 \pm 0.08$ \\
\hline 24 & L. thalamus & $1.08 \pm 0.12$ & $1.14 \pm 0.12$ & $1.04 \pm 0.08$ & $1.11 \pm 0.12$ & $0.95 \pm 0.24$ & $1.04 \pm 0.18$ & $1.25 \pm 0.15$ & $1.19 \pm 0.11$ \\
\hline 25 & L. V. sum lat. & $1.50 \pm 0.14$ & $1.52 \pm 0.15$ & $1.36 \pm 0.17$ & $1.39 \pm 0.18$ & $1.31 \pm 0.23$ & $1.29 \pm 0.03$ & $1.72 \pm 0.19$ & $1.69 \pm 0.12$ \\
\hline 26 & L. V. sum sup. & $1.18 \pm 0.15$ & $1.32 \pm 0.18$ & $1.36 \pm 0.03$ & $1.28 \pm 0.20$ & $1.24 \pm 028$ & $1.37 \pm 0.13$ & $1.52 \pm 0.19$ & $1.60 \pm 0.22$ \\
\hline 27 & R. fornix & $0.56 \pm 0.03$ & $0.55 \pm 0.09$ & $0.54 \pm 0.01$ & $0.51 \pm 0.08$ & $0.45 \pm 013$ & $0.48 \pm 0.04$ & $0.61 \pm 0.03$ & $0.51 \pm 0.05$ \\
\hline 28 & R. amygdala & $0.63 \pm 0.07$ & $0.63 \pm 0.08$ & $0.62 \pm 0.09$ & $0.55 \pm 0.07$ & $0.54 \pm 0.13$ & $0.53 \pm 0.04$ & $0.72 \pm 0.08$ & $0.58 \pm 0.06$ \\
\hline 29 & R. ant. comm. & $1.05 \pm 0.03$ & $1.12 \pm 0.11$ & $1.05 \pm 0.06$ & $1.02 \pm 0.14$ & $0.92 \pm 0.25$ & $1.03 \pm 0.16$ & $1.20 \pm 0.14$ & $1.22 \pm 0.11$ \\
\hline 30 & R. cerebellum & $0.36 \pm 0.07$ & $0.39 \pm 0.07$ & $0.38 \pm 0.09$ & $0.35 \pm 0.12$ & $0.33 \pm 0.13$ & $0.36 \pm 0.12$ & $0.43 \pm 0.10$ & $0.48 \pm 0.13$ \\
\hline 31 & R. corpus callosum & $1.84 \pm 0.21$ & $1.85 \pm 0.32$ & $1.80 \pm 0.09$ & $1.67 \pm 0.35$ & $1.60 \pm 0.44$ & $1.63 \pm 0.25$ & $2.08 \pm 0.23$ & $2.02 \pm 0.27$ \\
\hline 32 & R. fasci. retroflexus & $1.27 \pm 0.15$ & $1.32 \pm 0.12$ & $1.27 \pm 0.05$ & $1.27 \pm 0.13$ & $1.10 \pm 0.24$ & $1.15 \pm 0.22$ & $1.43 \pm 0.14$ & $1.36 \pm 0.11$ \\
\hline 33 & R. frontal lobe & $0.95 \pm 0.12$ & $0.92 \pm 0.13$ & $0.92 \pm 0.09$ & $0.86 \pm 0.18$ & $0.80 \pm 0.28$ & $0.81 \pm 0.24$ & $1.04 \pm 0.08$ & $1.09 \pm 0.12$ \\
\hline 34 & R. globus pallidus & $0.94 \pm 0.04$ & $1.03 \pm 0.09$ & $0.94 \pm 0.08$ & $0.94 \pm 0.10$ & $0.88 \pm 0.21$ & $0.98 \pm 0.09$ & $1.12 \pm 0.16$ & $1.11 \pm 0.09$ \\
\hline 35 & R. hippocampus & $1.68 \pm 0.16$ & $1.74 \pm 0.23$ & $1.67 \pm 0.06$ & $1.59 \pm 0.22$ & $1.52 \pm 0.38$ & $1.47 \pm 0.21$ & $1.92 \pm 0.20$ & $1.80 \pm 0.22$ \\
\hline 36 & R. hypothal. & $0.57 \pm 0.04$ & $0.58 \pm 0.08$ & $0.55 \pm 0.02$ & $0.54 \pm 0.08$ & $0.47 \pm 0.13$ & $0.51 \pm 0.05$ & $0.64 \pm 0.05$ & $0.56 \pm 0.05$ \\
\hline 37 & R. int. capsule & $0.87 \pm 0.05$ & $0.94 \pm 0.09$ & $0.87 \pm 0.09$ & $0.88 \pm 0.11$ & $0.83 \pm 0.21$ & $0.89 \pm 0.06$ & $1.04 \pm 0.14$ & $1.00 \pm 0.08$ \\
\hline 38 & R. mammilothal. tr. & $0.87 \pm 0.13$ & $0.92 \pm 0.14$ & $0.80 \pm 0.03$ & $0.88 \pm 0.13$ & $0.73 \pm 0.23$ & $0.78 \pm 0.12$ & $0.98 \pm 0.15$ & $0.93 \pm 0.09$ \\
\hline 39 & R. medulla & $0.02 \pm 0.01$ & $0.02 \pm 0.01$ & $0.02 \pm 0.01$ & $0.02 \pm 0.01$ & $0.02 \pm 0.01$ & $0.02 \pm 0.01$ & $0.02 \pm 0.01$ & $0.02 \pm 0.01$ \\
\hline 40 & R. midbrain & $1.62 \pm 0.10$ & $1.65 \pm 0.19$ & $1.60 \pm 0.16$ & $1.52 \pm 0.25$ & $1.45 \pm 0.31$ & $1.53 \pm 0.26$ & $1.77 \pm 0.18$ & $1.75 \pm 0.20$ \\
\hline 41 & R. occ. cortex & $0.97 \pm 0.16$ & $0.99 \pm 0.16$ & $0.91 \pm 0.09$ & $0.85 \pm 0.18$ & $0.79 \pm 0.17$ & $0.92 \pm 0.18$ & $1.06 \pm 0.15$ & $1.16 \pm 0.20$ \\
\hline 42 & R. olf. bulb & $1.07 \pm 0.11$ & $1.08 \pm 0.11$ & $1.01 \pm 0.07$ & $0.97 \pm 0.17$ & $0.86 \pm 0.30$ & $0.91 \pm 0.25$ & $1.14 \pm 0.17$ & $1.24 \pm 0.15$ \\
\hline 43 & R. optic nerve & $0.30 \pm 0.05$ & $0.33 \pm 0.09$ & $0.35 \pm 0.03$ & $0.26 \pm 0.05$ & $0.22 \pm 0.11$ & $0.25 \pm 0.09$ & $0.38 \pm 0.03$ & $0.35 \pm 0.04$ \\
\hline 44 & R. P-T cortex & $0.92 \pm 0.10$ & $0.90 \pm 0.18$ & $0.90 \pm 0.06$ & $0.80 \pm 0.19$ & $0.70 \pm 0.21$ & $0.78 \pm 0.12$ & $1.04 \pm 0.04$ & $1.01 \pm 0.11$ \\
\hline 45 & R. pons & $0.18 \pm 0.01$ & $0.19 \pm 0.01$ & $0.18 \pm 0.02$ & $0.18 \pm 0.00$ & $0.18 \pm 0.02$ & $0.16 \pm 0.02$ & $0.19 \pm 0.01$ & $0.19 \pm 0.02$ \\
\hline 46 & R. str. med. thal. & $0.82 \pm 0.04$ & $0.84 \pm 0.14$ & $0.85 \pm 0.09$ & $0.85 \pm 0.12$ & $0.78 \pm 0.07$ & $0.80 \pm 0.09$ & $0.69 \pm 0.16$ & $0.79 \pm 0.14$ \\
\hline 47 & R. striatum & $1.37 \pm 0.09$ & $1.41 \pm 0.17$ & $1.40 \pm 0.06$ & $1.27 \pm 0.17$ & $1.28 \pm 0.27$ & $1.31 \pm 0.08$ & $1.59 \pm 0.17$ & $1.59 \pm 0.15$ \\
\hline 48 & R. sub. region frontal & $0.77 \pm 0.03$ & $0.81 \pm 0.10$ & $0.82 \pm 0.07$ & $0.72 \pm 0.07$ & $0.66 \pm 0.16$ & $0.70 \pm 0.06$ & $0.89 \pm 0.09$ & $0.93 \pm 0.09$ \\
\hline 49 & R. sub. region (P-T) & $0.78 \pm 0.04$ & $0.85 \pm 0.09$ & $0.75 \pm 0.04$ & $0.75 \pm 0.08$ & $0.67 \pm 0.18$ & $0.76 \pm 0.09$ & $0.93 \pm 0.10$ & $0.87 \pm 0.08$ \\
\hline 50 & R. thalamus & $1.02 \pm 0.13$ & $1.09 \pm 0.12$ & $1.03 \pm 0.03$ & $1.06 \pm 0.13$ & $0.94 \pm 0.26$ & $0.96 \pm 0.15$ & $1.19 \pm 0.14$ & $1.14 \pm 0.12$ \\
\hline 51 & R. V. sum lat. & $1.26 \pm 0.11$ & $1.32 \pm 0.17$ & $1.24 \pm 0.18$ & $1.25 \pm 0.12$ & $1.17 \pm 0.25$ & $1.11 \pm 0.09$ & $1.50 \pm 0.18$ & $1.39 \pm 0.12$ \\
\hline 52 & R. V. sum sup. & $1.18 \pm 0.17$ & $1.31 \pm 0.19$ & $1.31 \pm 0.04$ & $1.26 \pm 0.23$ & $1.26 \pm 0.30$ & $1.32 \pm 0.10$ & $1.50 \pm 0.20$ & $1.52 \pm 0.24$ \\
\hline 53 & Third V. & $0.62 \pm 0.14$ & $0.78 \pm 0.10$ & $0.70 \pm 0.08$ & $0.78 \pm 0.12$ & $0.65 \pm 0.21$ & $0.78 \pm 0.04$ & $0.89 \pm 0.16$ & $0.87 \pm 0.13$ \\
\hline
\end{tabular}

ant., anterior; comm., commissure; fasci., fasciculus; hypothal., hypothalamus; int., internal; lat., lateral; mammilothal., mammilothalamic; occ., occipital; olf., olfactory; P-T, parietotemporal; str. med. thal., stria medullaris thalami; sub., subcortical; sup., superior; tr., tract; V., ventricle.

Terminal deoxynucleotidyl transferase-mediated dUTP nick-end labeling (TUNEL) assay. For the detection of apoptotic level of brain tissue sections preinjected with vehicle, PAM150, PAM500, and Itu1000, TUNEL staining was performed with the In situ Apoptosis Detection Kit (Takara MK500) according to the manufacturer's instructions. Briefly, the permeabilization buffer $(10 \mu \mathrm{l})$ was applied to each tissue section on a microscope slide for $5 \mathrm{~min}$. Then, the reaction mixture $(5 \mu \mathrm{l}$ of TdT enzyme $+45 \mu$ l of fluorescein labeling buffer) was applied to a slide and the samples were incubated in the incubator under $37^{\circ} \mathrm{C}$ for $90 \mathrm{~min}$. Incubated samples were washed two times with PBS for 10 min each time. Then, TUNEL-stained samples were incubated with Nissl stain
(NeuroTrace 530/615, N21482) for $20 \mathrm{~min}$ at room temperature. The samples with TUNEL staining were mounted with ProLong Diamond Antifade Mountant with DAPI (Thermo Fisher Scientific). The captured images were analyzed by counting cells with TUNEL signal per cells with positive DAPI/Nissl signal.

For the detection of apoptosis in neuron cultures under PAM (500, $2500 \mathrm{ng} / \mathrm{ml})$, Itu (1000 ng/ml), and staurosporine (STS) $(0.75 \mu \mathrm{M})$ (Cayman Chemical 81590), TUNEL staining was performed according to the manufacturer's instructions. STS was used as an apoptosis-inducing agent (positive control). The permeabilization buffer $(100 \mu \mathrm{l})$ was applied to fixed neuron cultures on coverslips for $5 \mathrm{~min}$. After rinsing with 
A
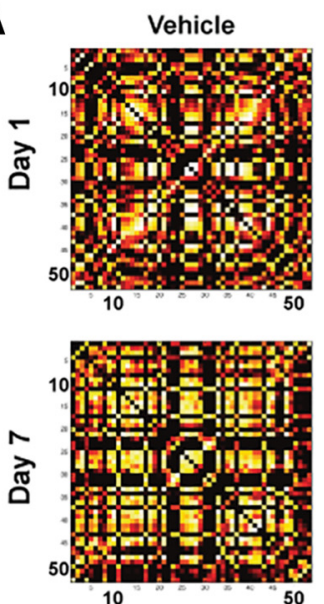

B
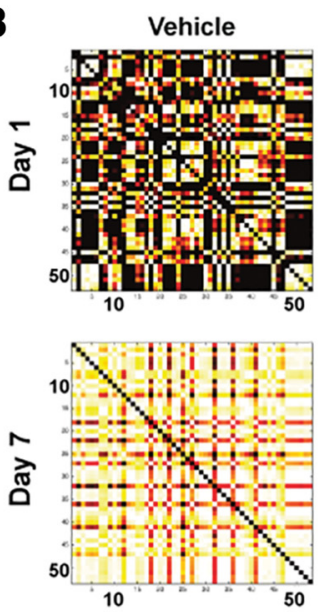

PAM (150)
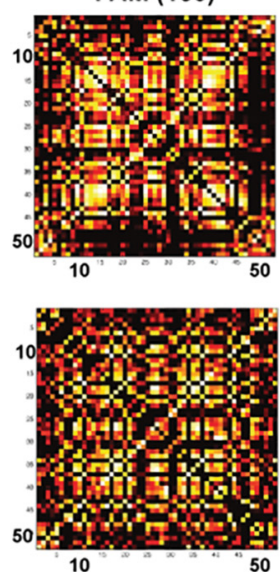

PAM (150)
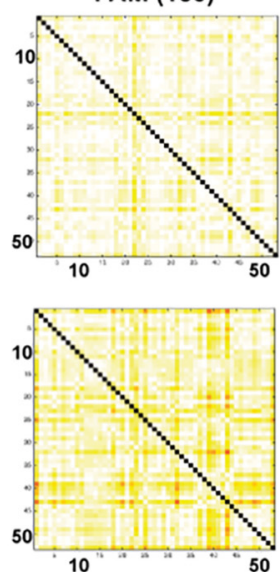

$\operatorname{PAM}(500)$
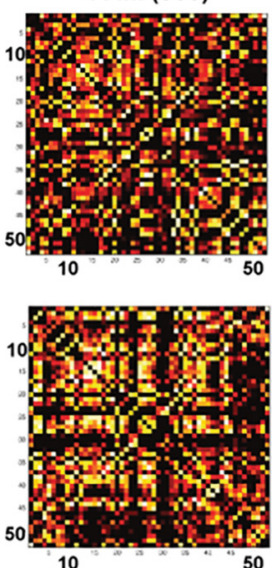

10

PAM (500)
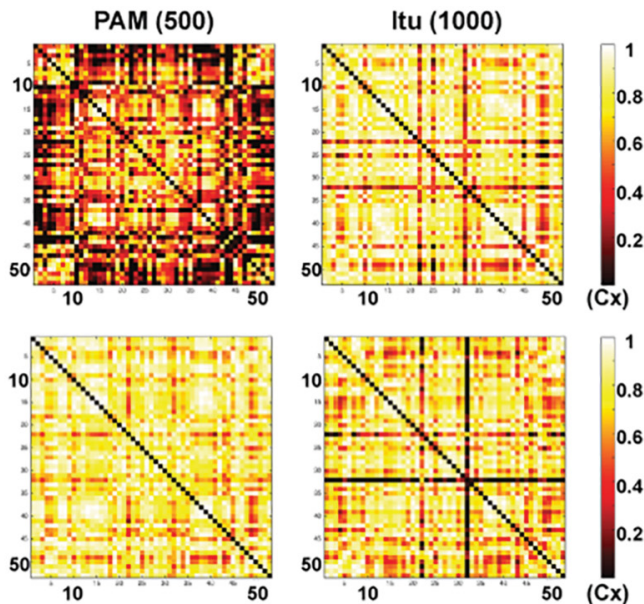

Itu (1000)
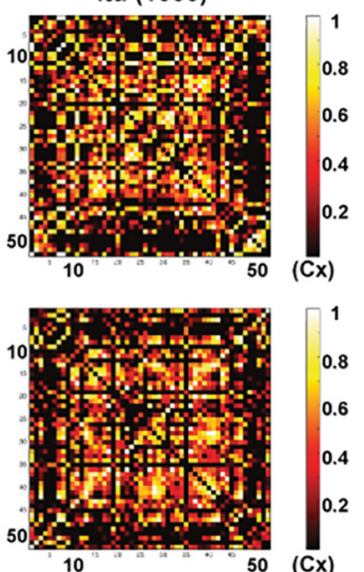

Itu $(1000)$

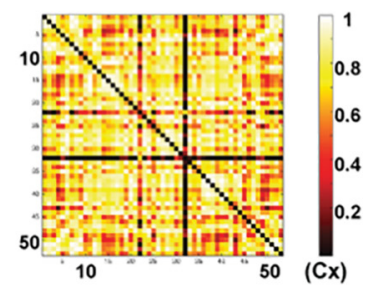

Figure 2. Correlation matrices from $\left[{ }^{18} \mathrm{~F}\right] \mathrm{FDG}$ PET and $\left[{ }^{18} \mathrm{~F}\right] \mathrm{FMZ}$ PET. $A$, Interregional correlation matrices $(53 \times 53)$ were obtained from calculations of correlations between two nodes in the mouse brain from $\left[{ }^{18} \mathrm{~F}\right]$ FDG PET images of vehicle, PAM, and Itu groups. Each node was predefined by $53 \mathrm{VOls}$ (Table 1). Correlations $(\mathrm{Cx})$ between two nodes $(i, j)$ in the brain at day 1 and day 7 for each group were defined as $\mathrm{Cx}(i, j)=1: r(i, j)$, where $r$ is a Pearson's correlation coefficient. The comparison of correlation matrices between vehicle and test groups were later performed by permutation test ( 5000 times for each of $53 \mathrm{~V}$ Ols) to determine statistical differences of metabolic correlation ( $p<0.005$ vs vehicle group) (Fig. $3 A)$. $\boldsymbol{B}$, Interregional correlation matrices ( $53 \times 53$ ) were obtained from calculations of correlations between two nodes in the mouse brain from $\left[{ }^{18} \mathrm{~F}\right]$ FMZ PET images of vehicle, PAM, and ltu groups. Each node was predefined by $53 \mathrm{~V}$ VIs (Table 1 ). Correlations $(\mathrm{Cx})$ between two nodes $(i, j)$ in the brain at day 1 and day 7 for each group were defined as $\mathrm{Cx}(i, j)=1: r(i, j)$, where $r$ is the Pearson's correlation coefficient. The comparison of correlation matrices between vehicle and test groups were later performed by permutation test ( 5000 times for each of $53 \mathrm{~V}$ VIs) to determine statistical differences of neuronal correlation $(p<0.005 \mathrm{vs} \mathrm{vehicle}$ group) (Fig. 3B).

PBS, the reaction mixture $(5 \mu \mathrm{l}$ of TdT enzyme $+45 \mu \mathrm{l}$ of fluorescein labeling buffer) was applied to each coverslip and the samples were incubated in the incubator under $37^{\circ} \mathrm{C}$ for $90 \mathrm{~min}$. Incubated samples were washed with PBS for 10 min twice. Then, TUNEL-stained samples were incubated with Alexa Fluor 555 phalloidin (Cell Signaling Technology 8953 ) for $15 \mathrm{~min}$ at room temperature. The samples with TUNEL staining were mounted with ProLong Diamond Antifade Mountant with DAPI (Thermo Fisher Scientific). The captured images were analyzed by counting cells with TUNEL signal per cells with positive DAPI/actin signal.

Western blot. For analyzing protein expressions from tissue sections at day 1 and day 7 ( $n=3$ per each group), mice were decapitated under isoflurane anesthesia and their brains were collected and rapidly frozen on dry ice. The brain area in the CA1 next to the injection site on the left side of brain was scraped into $200 \mu \mathrm{l}$ of lysis solution (Ambion). The Protein concentration in the samples was measured with Bradford reagent (Bio-Rad). Samples were stored at $-80^{\circ} \mathrm{C}$ until needed. To prepare samples for Western blotting, $100 \mu \mathrm{g}$ of total protein was precipitated from lysis buffer with $90 \%(\mathrm{v} / \mathrm{v})$ methanol. The protein was then pelleted by centrifugation $(10,000 \times g, 10 \mathrm{~min}$ at room temperature), washed with $1 \mathrm{ml}$ of $90 \%$ methanol, dried, and dissolved in SDS sample buffer at a final concentration of $0.5 \mathrm{mg} / \mathrm{ml}$. The samples were run in 26-well
Criterion cassettes (Bio-Rad) and proteins were transferred onto Immobilon-P membrane (Millipore). Transferred membranes were blocked with $5 \%$ nonfat dry milk in $1 \times$ TBST buffer containing $20 \mathrm{~mm}$ Tris- $\mathrm{HCl}$, pH 7.4, $150 \mathrm{~mm} \mathrm{NaCl}$, and 0.01\% Tween 20.

For analyzing protein expressions from cultured neurons $(n=3$ per each group), cells were lysed using N-PER reagent (Thermo Fisher Scientific) supplemented with Halt Protease Inhibitor Mixture (Thermo Fisher Scientific). The protein lysates were centrifuged at 12,000 rpm for $10 \mathrm{~min}$ at $4^{\circ} \mathrm{C}$. The protein concentration of harvested supernatant was measured using the BCA protein assay kit (Thermo Fisher Scientific). Equal amounts of proteins were loaded into 10\% Nu-PAGE gel and separated electrophoretically at $120 \mathrm{~V}$ and transferred onto PVDF membrane (Invitrogen). Transferred membranes were blocked with 5\% nonfat dry milk in $1 \times$ TBST buffer containing $20 \mathrm{~mm}$ Tris- $\mathrm{HCl}$, $\mathrm{pH} 7.4,150$ $\mathrm{mm} \mathrm{NaCl}$, and $0.01 \%$ Tween 20 .

The blocked membranes from both in vivo and in vitro experiments were probed with following primary antibodies: guinea pig polyclonal anti-VGluT1 (Synaptic Systems, 1:1000) and rabbit polyclonal antiHomer1 (Synaptic Systems, 1:3000), and mouse monoclonal anti$\beta$-actin (Sigma-Aldrich, 1:5000). The anti- $\beta$-actin antibody served as a loading control. The secondary antibodies used were horseradish peroxidase-conjugated anti-guinea pig, anti-rabbit, and anti-mouse an- 
tibodies (Jackson ImmunoResearch Laboratories). All secondary antibodies were incubated at room temperature for $90 \mathrm{~min}$. The incubated membrane was developed using an enhanced chemiluminescence kit (Thermo Fisher Scientific). Membranes were exposed to autoradiography films (Santa Cruz Biotechnology) and developed on a developer (Konica Minolta SRX-101A). The blots from three replicate experiments were quantified by densitometric analysis using ImageJ.

Experimental design and statistical analysis. The current study consisted of whole-brain imaging (i.e., PET imaging) ( $n=7$ per group), in vivo assays (IHC, $n=4$; TUNEL, $n=3$, Western blot, $n=3$ ), and in vitro assays (ICC, $n=3$ or 4 ; TUNEL, $n=3$, Western blot, $n=3$ ). For electrophysiology, fEPSP ( $n=10$ per group) and LTP ( $n=5$ vehicle, 6 PAM, 4 Itu) were measured for brain slices and the frequency and amplitude of mEPSCs ( $n=7$ to 13 ) were measured for neuron cultures. All treatments were repeated independently for randomly chosen groups. All analyses except Western blot assay were conducted in a blinded manner. The identity of the condition was not revealed until the completion of analysis. Data are represented as mean \pm SD or SEM as described in the figure legends. To determine statistical significance, one-way ANOVA was performed followed by post hoc analysis with Tukey's multiple-comparisons test using SigmaPlot 12.5 (Systat Software). For the comparison of amplitude and interevent interval of mEPSC in cumulative probability, the K-S test was performed. The level of statistical significance was set at ${ }^{*} p<0.05$ and ${ }^{* *} p<0.001$ for all experiments except PET analysis, where $p<0.005$ was used as the statistical threshold.

The power analysis was also performed to determine whether the sample size yielded sufficient power to conclude a statistical significance using SigmaPlot 12.5 (Systat Software). A result with a power $<50 \%(0.5<, \alpha=0.05)$ was not considered statistically significant even when the $p$-value was $<0.05$.

\section{Results}

Abnormal metabolic activity induced by PAM recovers in $7 \mathrm{~d}$, whereas abnormal neuronal integrity partially recovers

To investigate functional damage in the brain induced by bacterial lipopeptides, we performed PET imaging of mice brains injected with PAM150 and PAM500 into the CA1 of the left hippocampi. We also injected Itu1000 as another bacterial lipopeptide model that has been reported to be nonvirulent and nontoxic to cells (Klich et al., 1994; Singh and Cameotra, 2004). For PET imaging, we used $\left[{ }^{18} \mathrm{~F}\right]$ FDG and $\left[{ }^{18} \mathrm{~F}\right]$ FMZ to examine metabolic activity and neuronal integrity, respectively, of mice brains at day 1 and 7 after injection. At day 1, analysis of FDG PET images revealed that PAM500 decreased metabolic activity in the injection site (i.e., hippocampus) and bilateral striatum $(p<$ 0.005 vs vehicle), whereas PAM150 did not induce any abnormal metabolic activity in the brain (Fig. 1A). Itu1000 also altered metabolic activity in the ipsilateral hippocampus with less abnormality than PAM500. At day 7, however, the abnormal metabolic activity induced by PAM500 and Itu1000 at day 1 recovered to the level of the vehicle groups (Fig. 1A).

Analysis of FMZ PET images revealed that both PAM and Itu altered $\mathrm{BP}_{\mathrm{ND}}$ at day 1 after injection ( $p<0.005$ vs vehicle) (Fig. $1 B)$. PAM500 induced a reduction in $\mathrm{FMZ} \mathrm{BP}_{\mathrm{ND}}$ in the areas including bilateral hippocampus, lateral cortex, and thalamus, whereas PAM150 and Itu1000 induced a reduction in FMZ BP mainly in lateral cortex and hippocampus (Table 1). The abnormal neuronal activity induced by PAM500 and Itu1000 in hippocampus and lateral cortex and hippocampus remained until day 7 after injection (Fig. 1B).

Neuronal network impaired by PAM and Itu is aggravated in a different pattern $7 \mathrm{~d}$ after injection

To determine abnormal functional networks in the brains injected with PAM150, PAM500, and Itu1000, we constructed in-
Table 2. VOls $(n=53)$ in the mouse brain used to determine altered correlation between two nodes in the brain from analysis of PET imaging

\begin{tabular}{|c|c|}
\hline Region\# & Region name \\
\hline 1 & left_amygdala \\
\hline 2 & left_frontal_cortex \\
\hline 3 & left_optic_nerve \\
\hline 4 & left_anterior_commissure \\
\hline 5 & left_cerebellum \\
\hline 6 & left_corpus_callosum \\
\hline 7 & left_fasciculus_retroflexus \\
\hline 8 & left_fornix \\
\hline 9 & left_globus_pallidus \\
\hline 10 & left_hippocampus \\
\hline 11 & left_hypothalamus \\
\hline 12 & left_internal_capsule \\
\hline 13 & left_mammilothalamic_tract \\
\hline 14 & left_medulla \\
\hline 15 & left_midbrain \\
\hline 16 & left_occipital_cortex \\
\hline 17 & left_olfactory_bulb \\
\hline 18 & left_parietal_temporal_cortex \\
\hline 19 & left_pons \\
\hline 20 & left_stria_medullaris_thalami \\
\hline 21 & left_striatum \\
\hline 22 & left_subcortical_region_parieto_temporal \\
\hline 23 & left_subcortical_region_frontal \\
\hline 24 & left_thalamus \\
\hline 25 & left_ventricle_sum_lateral \\
\hline 26 & left_ventricle_sum_superior \\
\hline 27 & right_fornix \\
\hline 28 & right_amygdala \\
\hline 29 & right_anterior_commissure \\
\hline 30 & right_cerebellum \\
\hline 31 & right_corpus_callosum \\
\hline 32 & right_fasciculus_retroflexus \\
\hline 33 & right_frontal_cortex \\
\hline 34 & right_globus_pallidus \\
\hline 35 & right_hippocampus \\
\hline 36 & right_hypothalamus \\
\hline 37 & right_internal_capsule \\
\hline 38 & right_mammilothalamic_tract \\
\hline 39 & right_medulla \\
\hline 40 & right_midbrain \\
\hline 41 & right_occipital_cortex \\
\hline 42 & right_olfactory_bulb \\
\hline 43 & right_optic_nerve \\
\hline 44 & right_parietal_temporal_cortex \\
\hline 45 & right_pons \\
\hline 46 & right_stria_medullaris_thalami \\
\hline 47 & right_striatum \\
\hline 48 & right_subcortical_region_frontal \\
\hline 49 & right_subcortical_region_parieto_temopora \\
\hline 50 & right_thalamus \\
\hline 51 & right_ventricle_sum_lateral \\
\hline 52 & right_ventricle_sum_superior \\
\hline 53 & third_ventricle \\
\hline
\end{tabular}

terregional correlation matrices for each group (Fig. 2) by extracting the mean count of $\left[{ }^{18} \mathrm{~F}\right]$ FDG uptake and the mean $\mathrm{BP}_{\mathrm{ND}}$ of $\left[{ }^{18} \mathrm{~F}\right] \mathrm{FMZ}$ for evaluating metabolic and neuronal correlations between various brain regions, respectively (Choi et al., 2014). The interregional correlation matrices used 53 anatomical VOIs in the mouse brain to examine the correlation between two nodes in the brain (Dorr et al., 2007) (Table 2). The differences of metabolic correlation between vehicle and other groups revealed that both PAM500 and Itu1000 altered correlations (Cx) between 


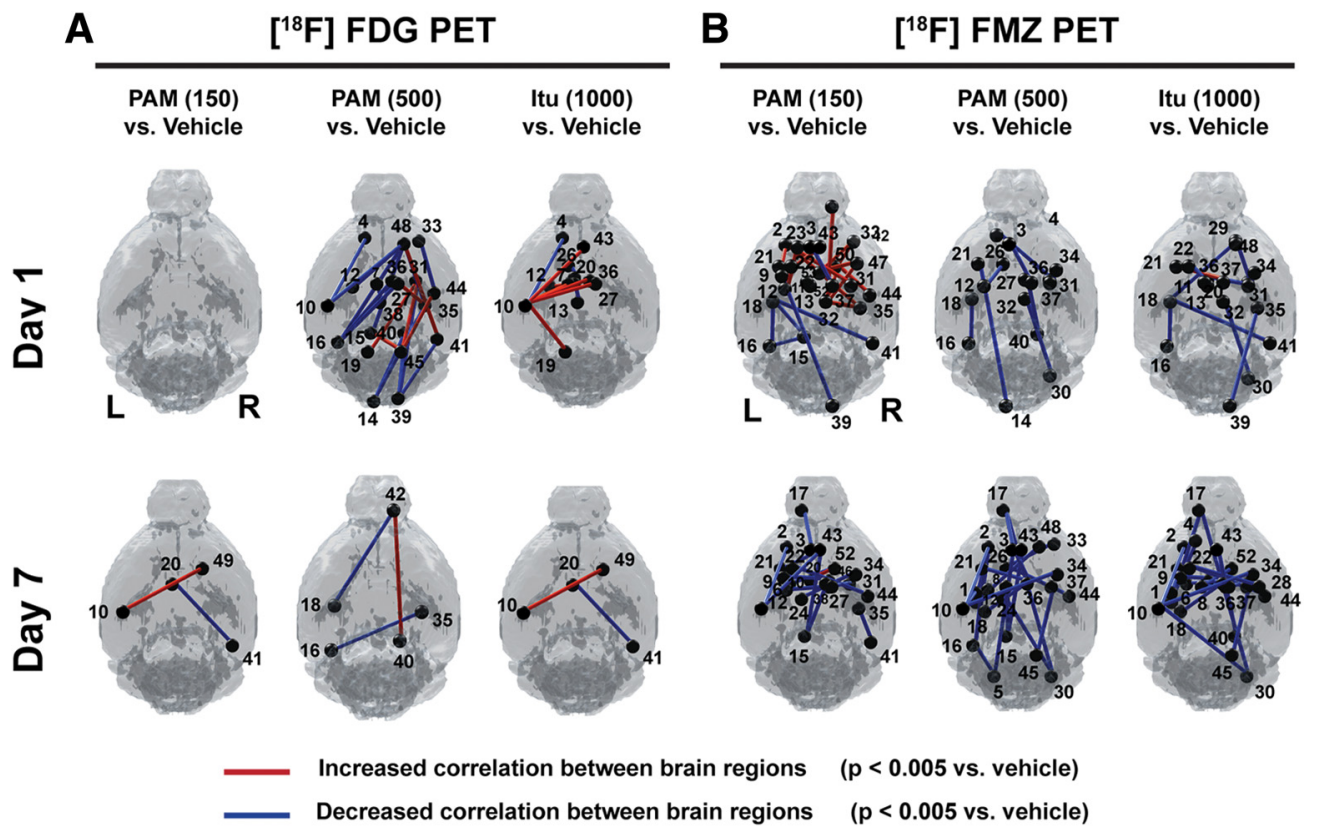

Figure 3. Functional correlation map in different regions of mice brains for $7 \mathrm{~d}$ following stereotactic injection of PAM and Itu into the left CA1. Numbering information for brain nodes (VOIs) is available in Table 1. A, Altered metabolic correlation in the brain $(p<0.005$ vs vehicle) was determined by the correlation matrix (Fig. $2 A)$ and permutation test. PAM500 showed decreased metabolic correlation between ipsilateral hippocampus and various VOIs at day 1 after injection. Itu 1000 showed increased correlation between several pairwise V0Is at day 1 after injection. Majority of abnormal metabolic correlation recovered at day 7 after injection. $\boldsymbol{B}$, Altered neuronal correlation $(p<0.005$ vs vehicle) was determined by the correlation matrix (Fig. 2B) and permutation test. PAM150 showed increased neuronal correlation between ipsilateral hippocampus and several V0Is, whereas PAM500 and Itu1000 showed decreased neuronal correlation between many pairwise VOls at day 1 after injection. At day 7 after injection, all test groups had more pairwise V0ls with decreased neuronal correlation. Red lines and blue lines indicate increased and decreased correlation between two brain regions of $53 \mathrm{~V}$ Ols, respectively ( $p<0.005$ vs vehicle, $n=7$ mice per each group).

Table 3. Altered correlation between various brain regions in $\left[{ }^{18} \mathrm{~F}\right] \mathrm{FDG}$ PET/CT

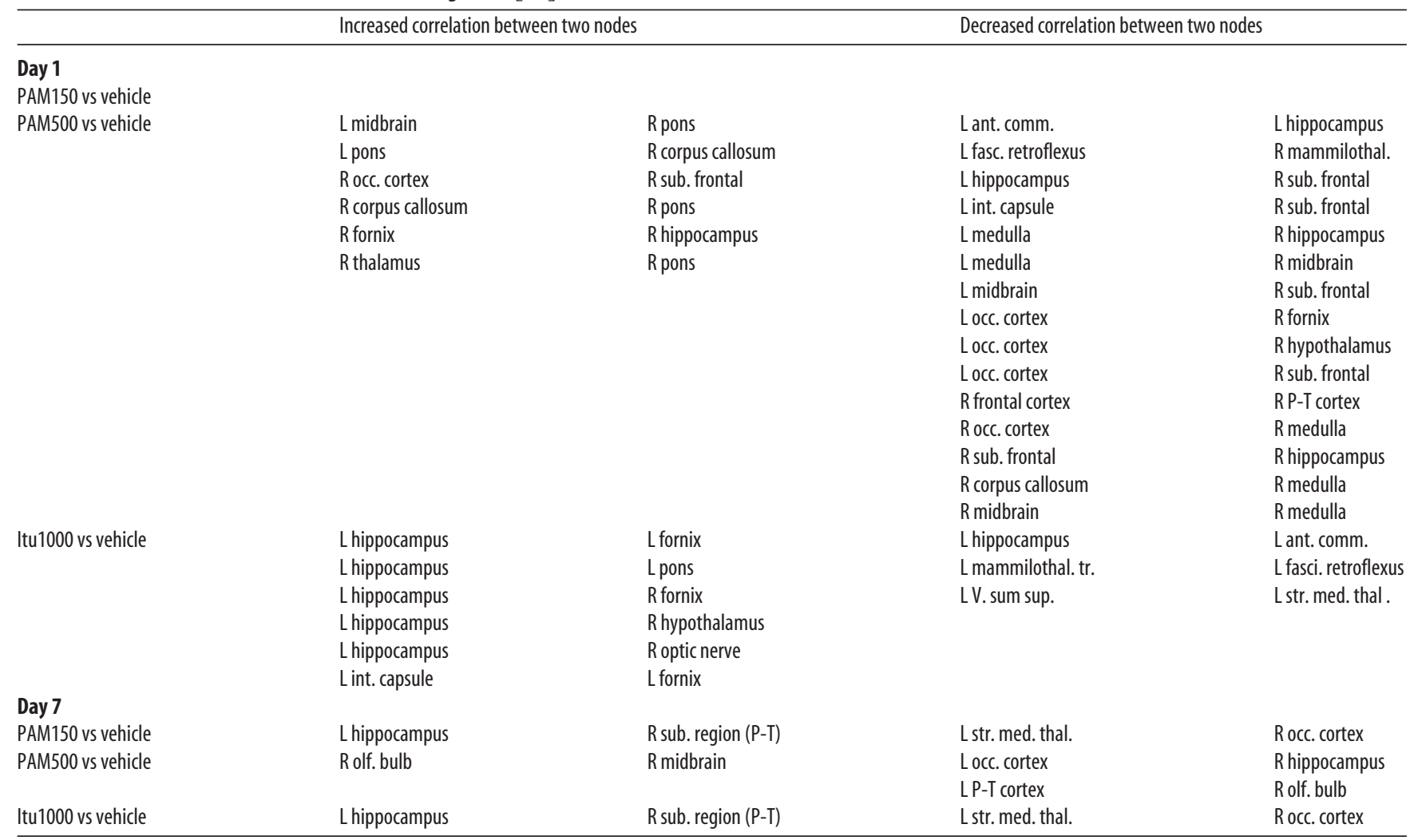

From the comparison of the correlation matrices from [ $\left.{ }^{18} \mathrm{~F}\right] \mathrm{FDG}$ PET analysis (Figure $2 A$ ), we determined altered metabolic correlation between vehicle and test groups $(p<0.005)$.

ant., anterior; comm., commissure; fasci., fasciulus; hypothal., hypothalamus; int., internal; lat., lateral; mammilothal., mammilothalamic; occ., occipital; olf., olfactory; P-T, parietotemporal; str. med. thal., stria medullaris thalami; sub., subcortical; sup., superior; tr., tract; $V_{\text {., ventricle. }}$ 
Table 4. Altered correlation between various brain regions in $\left[{ }^{18} \mathrm{~F}\right] \mathrm{FMZ}$ PET/CT

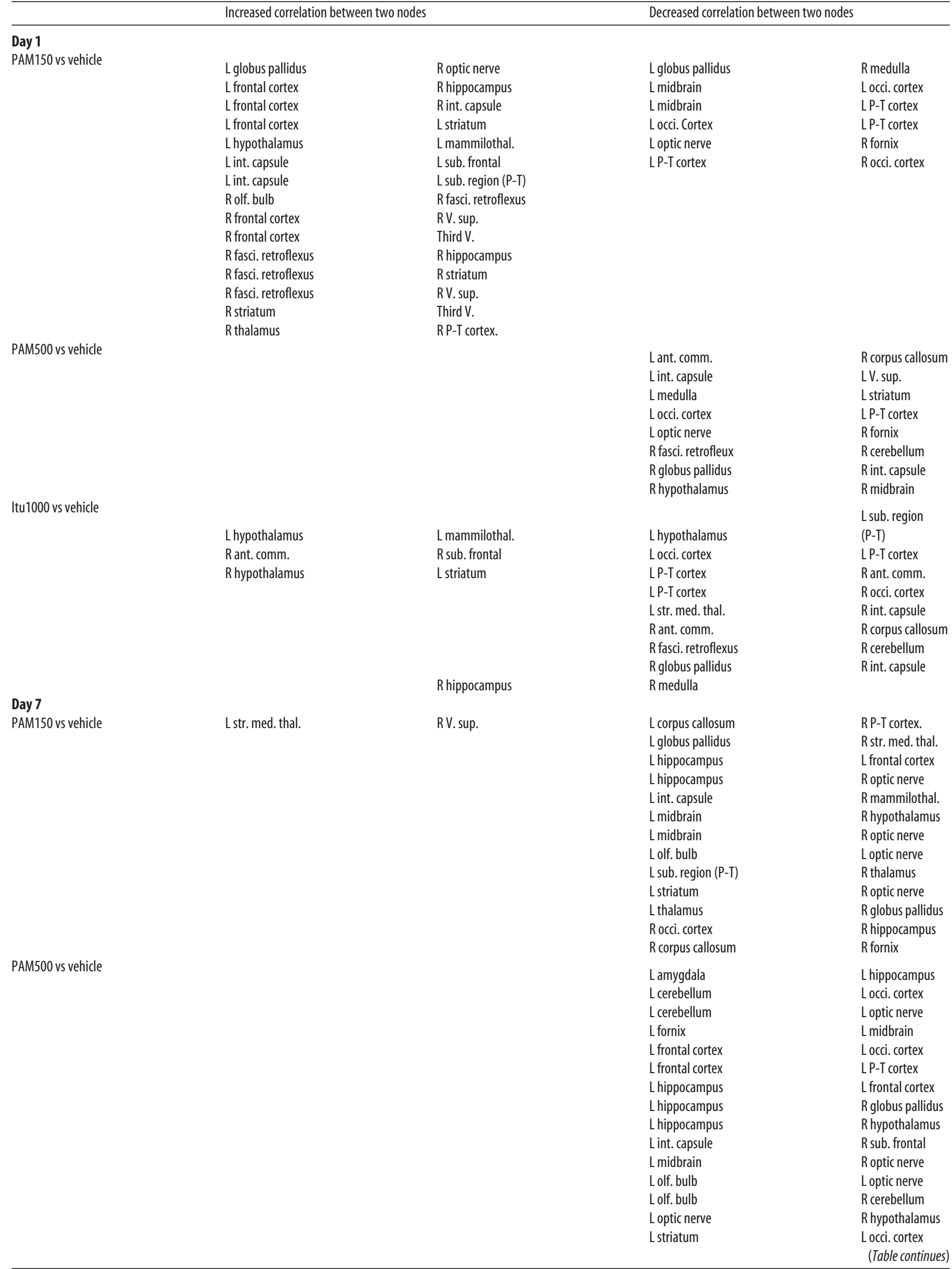




\begin{tabular}{|c|c|c|c|}
\hline & \multirow[t]{6}{*}{ Increased correlation between two nodes } & \multicolumn{2}{|c|}{ Decreased correlation between two nodes } \\
\hline & & L striatum & R hypothalamus \\
\hline & & L thalamus & R cerebellum \\
\hline & & LV. sup. & R frontal cortex \\
\hline & & LV.sup & RP-T cortex \\
\hline & & R int. capsule & Rpons \\
\hline \multirow[t]{18}{*}{ Itu1000 vs vehicle } & & Lamygdala & L striatum \\
\hline & & Lant. comm. & LP-T cortex \\
\hline & & L corpus callosum & R hypothalamus \\
\hline & & L fornix & R fornix \\
\hline & & L fornix & RV.sup \\
\hline & & L frontal cortex & L hippocampus \\
\hline & & L globus pallidus & RP-T cortex \\
\hline & & L hippocampus & R globus pallidus \\
\hline & & L hippocampus & R cerebellum \\
\hline & & L hippocampus & R hypothalamus \\
\hline & & Lolf. bulb & LP-T cortex \\
\hline & & L olf. bulb & R cerebellum \\
\hline & & L str. med. thal. & $\mathrm{R}$ thalamus \\
\hline & & L sub. region (P-T) & R amygdala \\
\hline & & R fornix & $\mathrm{R}$ corpus callosum \\
\hline & & R hypothalamus & R midbrain \\
\hline & & R hypothalamus & Roptic nerve \\
\hline & & Rint. capsule & Rpons \\
\hline
\end{tabular}

From the comparison of the correlation matrices from $\left[{ }^{18} \mathrm{~F}\right] \mathrm{FMZ}$ PET analysis (Figure $2 B$ ), we determined altered synaptic correlation between vehicle and test groups ( $p<0.005$ ).

ant., anterior; comm., commissure; fasci., fasciulus; hypothal., hypothalamus; int., internal; lat., lateral; mammilothal., mammilothalamic; occ., occipital; olf., olfactory; P-T, parietotemporal; str. med. thal., stria medullaris thalami; sub., subcortical; sup., superior; tr., tract; V., ventricle.

the pairwise VOIs shown in Figure $3 A$ and Table 3 at day 1 after injection. Interestingly, Itu1000 increased metabolic correlation (i.e., shorter correlations vs vehicle) between ipsilateral hippocampus and various VOIs, whereas PAM500 decreased metabolic correlation (i.e., longer correlations vs vehicle) between bilateral hippocampus and various VOIs $(p<0.005$ vs vehicle, permutation 5000). However, the abnormal metabolic correlation at day 1 after injection of PAM500 and Itu 1000 recovered and returned to the level of vehicle group in 7 d (Fig. 3A, Table 3).

The differences of neuronal correlation between vehicle and other groups revealed that all groups including PAM150, PAM500, and Itu1000 Cx between the pairwise VOIs shown in Figure $3 B$ and Table 4 at day 1 after injection. PAM150 increased the correlation in the pairwise VOIs involving contralateral hippocampus and decreased correlation in the VOIs on the injection side (i.e., left hemisphere). Both PAM500 and Itu1000 induced longer correlations (i.e., decreased or weaker correlation) between various pairwise VOIs $(p<0.005$ vs vehicle, permutation 5000). In contrast to the results of metabolic network from FDG PET analysis, the abnormal neuronal network by PAM150, PAM500, and Itu1000 at day 1 after injection did not recover in $7 \mathrm{~d}$. Rather, all groups showed aggravated networks in VOIs including amygdala, fornix, hippocampus, olfactory bulb, and thalamus at day 7 after injection. The majority of impaired networks induced by PAM500 involved ipsilateral nodes, whereas Itu1000-induced damage involved bilateral nodes (Fig. 3B, Table 4).

\section{PAM decreases both presynaptic and postsynaptic density in} hippocampal region at day 7 after injection

To determine whether the results of PET imaging that showed the abnormal neuronal integrity and network involve synaptic alteration, we measured synaptic density in brain tissue slices at day 1 and day 7 following stereotactic injection of PAM and Itu (Fig. 4).
Because FMZ PET analysis revealed that the abnormal neuronal activity and network induced by PAM and Itu involves brain regions of cortex (Ctx), hippocampus (HP), and lateral cortex and hippocampus (EC/HP), we measured presynaptic (VGluT1) and postsynaptic (Homer1) density in Ctx, HP, and EC/HP and compared synaptic density among groups (vehicle, PAM150, PAM500, and Itu1000) and between time courses (day 1 and day 7 ). The measurement of integrated density of fluorescent intensity from VGluT1 (see Materials and Methods) showed that PAM500 significantly decreased the density of fluorescent signal in $\mathrm{HP}(p=0.016$ vs vehicle) and $\mathrm{EC} / \mathrm{HP}(p=0.012$ vs vehicle), whereas Itu 1000 significantly decreased the density of fluorescent signal in $\mathrm{HP}(p=0.032$ vs vehicle $)$ and $\mathrm{Ctx}$ ( $p=0.047$ vs vehicle $)$ (Fig. $4 B$ ). With respect to the time courses, PAM500 significantly decreased the density of fluorescent signal in EC/HP at day 7 ( $p=$ 0.007 vs PAM500 Day 1 $_{1}$ ), whereas Itu1000 significantly decreased the density of fluorescent signal in Ctx over time $(p=0.006$ vs Itu $\left.1000_{\text {Day } 1}\right)$. The measurement of integrated density of fluorescent intensity from Homer1 showed that PAM500 significantly decreased the density of fluorescent signal in HP ( $p=0.036$ vs vehicle) (Fig. $4 C$ ). Itu1000 also decreased the density of fluorescent signal in HP, but the difference was not significant $(p=0.05$ vs vehicle). Although both PAM500 and Itu1000 decreased presynaptic and postsynaptic density in $\mathrm{HP}$, the expression level of VGluT1 or Homer 1 in HP was not altered at either day 1 or day 7 after injection (Fig. 4E).

For further quantification of synaptic loss at day 7 after PAM and Itu injection, we counted the number of presynaptic (VGluT1) and postsynaptic (Homer1) puncta per length of dendrite in the Ctx, HP (stratum radiatum), and EC/HP region (Fig. $5)$. The measurements showed that PAM500 decreased the number of presynaptic puncta in all regions $\left(p_{\mathrm{Ctx}}=0.0264, p_{\mathrm{HP}}=\right.$ $0.0015, p_{\mathrm{EC} / \mathrm{HP}}=0.014$ vs vehicle $)$ and the number of postsynaptic puncta in Ctx $(p=0.0363$ vs vehicle $)$ and $\mathrm{HP}(p=0.0459$ vs 
A
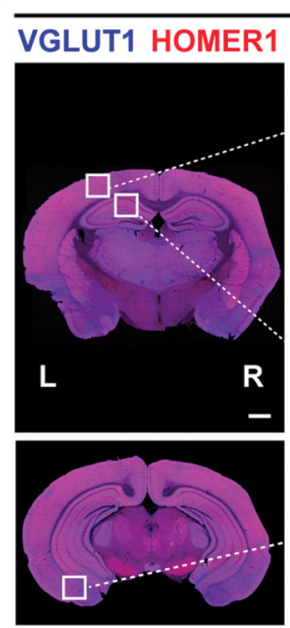

Vehicle (D7)
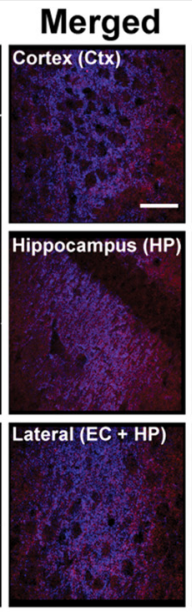
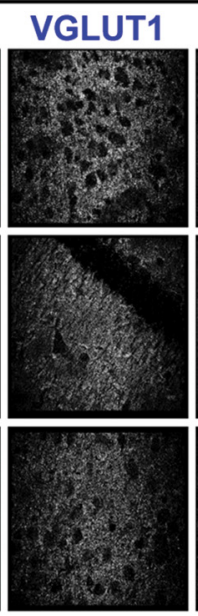

VGLUT1

B

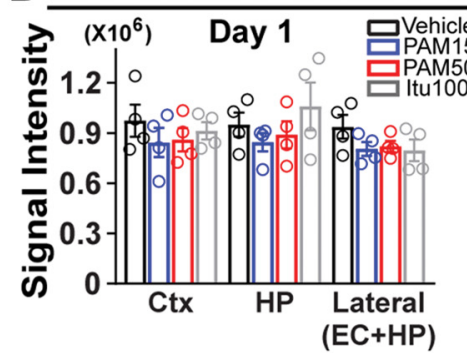

C

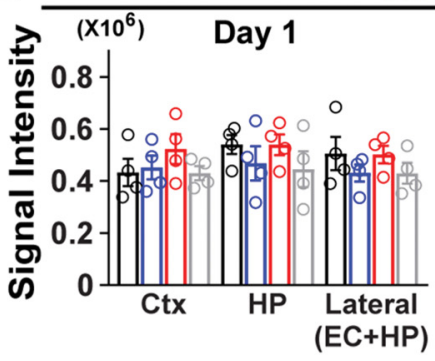

HOMER1
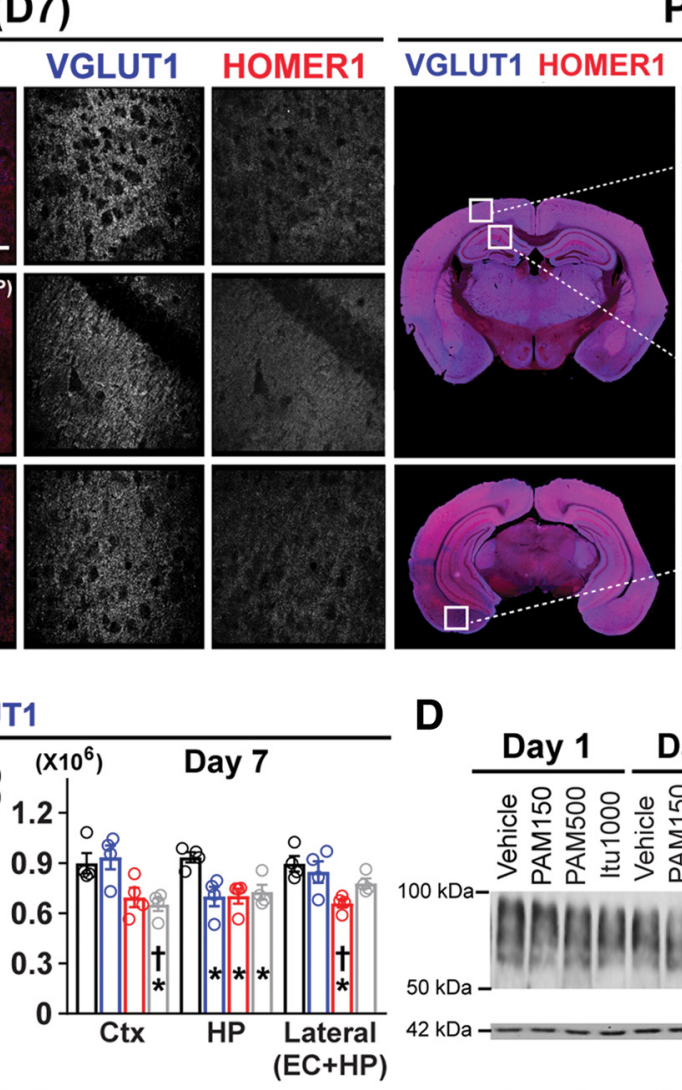

PAM500 (D7)
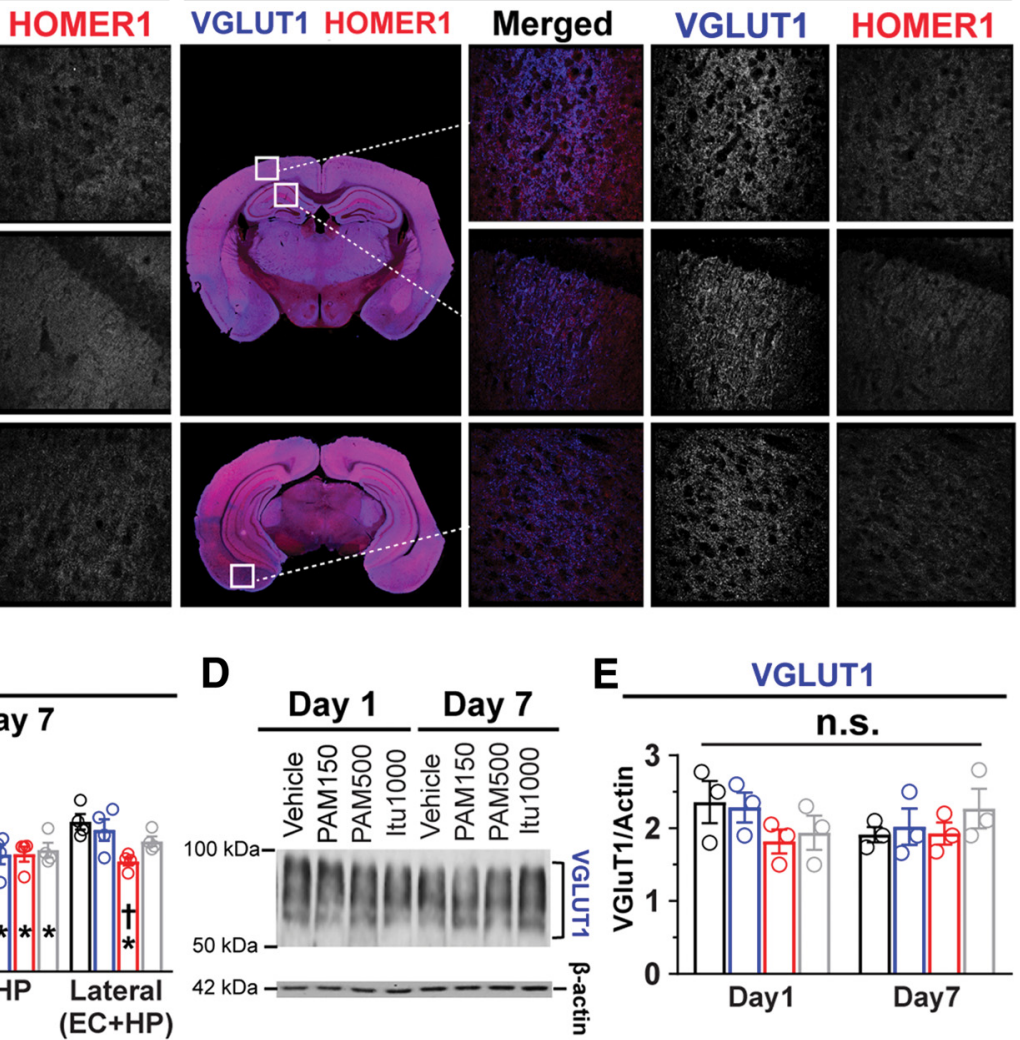

$E$

VGLUT1

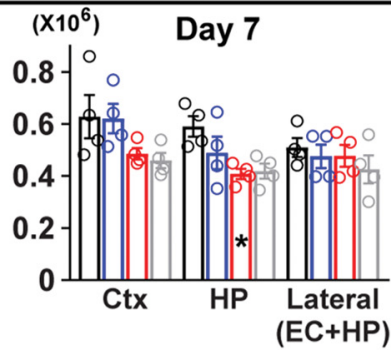

Day $1 \quad$ Day 7
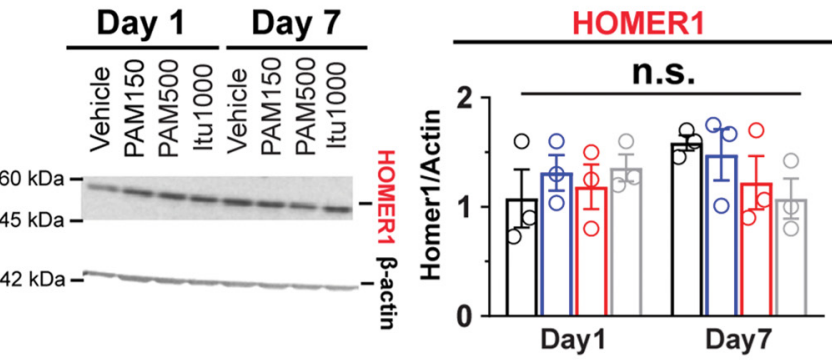

Figure 4. Effect of PAM on synaptic density in brain tissues. $\boldsymbol{A}$, Fluorescent images of brain sections stained with anti-VGluT1 (blue) and anti-Homer1 (red) at day 7 after injection of vehicle (left) and PAM500 (right). Boxes in the whole-brain section (scale bar, $500 \mu \mathrm{m}$ ) indicate the regions (Ctx, HP, and lateral EC, scale bar, $50 \mu \mathrm{m}$ ) where the integrated density of fluorescent intensity from presynapses (VGluT1) and postsynapses (Homer1) was measured (see Materials and Methods). B, Quantification of the integrated density of fluorescent intensity from VGluT1 at day 1 and day 7 after injection. Compared with vehicle, PAM150, PAM500, and Itu1000 significantly decreased the signal intensity of VGluT1 in the HP region at day7 after injection ( ${ }^{*} p<0.05$ vs vehicle). Compared with analysis at day 1, PAM500 significantly decreased the signal intensity of VGluT1 in the EC region ( $t p<0.05$ vs PAM500 ${ }_{\text {Day1-Lateral }}$ ) and Itu significantly decreased the signal intensity of VGluT1 in the $\mathrm{Ctx}\left(\mathrm{t} p<0.05 \mathrm{vs}\right.$ Itu1000 ${ }_{\text {Day } 1-\mathrm{Ctx}}$ ). All data are presented as mean $\pm \mathrm{SEM}$ of $n=4$ independent experiments. C, Quantification of the integrated density of fluorescent intensity from Homer1 at day 1 and day 7 after injection. Compared with vehicle, only PAM500 significantly decreased the signal intensity of Homer 1 in the HP region at day 7 after injection ( ${ }^{*} p<0.05$ vs vehicle). All data are presented as mean \pm SEM of $n=4$ independent experiments. D, Representative band images of Western blotting of VGluT1 and Homer 1 proteins from the HP region at day 1 and day 7 after injections. $\boldsymbol{E}$, Level of synaptic protein expressions was not altered by varied concentrations of PAM and Itu or by the time course (day 1 and day 7$)(p>0.05)$. Data are presented as mean \pm SEM of $n=3$ independent experiments.

vehicle). Itu1000 also decreased the number of presynaptic puncta in $\operatorname{Ctx}(p=0.0445$ vs vehicle) and $\operatorname{HP}$ ( $p=0.0066$ vs vehicle $)$ and the number of postsynaptic puncta only in $\mathrm{HP}(p=0.0231$ vs vehicle). These results are comparable to those from the measurement of integrated density of fluorescent intensity from VGluT1 and Homer1 in Ctx, HP, and EC/HP. Because the synaptic loss in HP (stratum radiatum) was more severe compared with other regions, we also measured the synaptic density of stratum oriens and stratum lacunosum moleculare in in HP, which are the areas in vicinity of stratum radiatum that we analyzed in HP. Unexpectedly, PAM increased the number of presynaptic ( $p=0.215$ vs vehicle) and postsynaptic ( $p=0.0343$ vs vehicle) puncta and Itu increased presynaptic puncta ( $p=0.0003$ vs vehicle) (Fig. 6). This result sug- gests that PAM or Itu challenges in the brain may have caused a local reorganization of synaptic distributions.

PAM alters synaptic transmission and plasticity in hippocampus

To assay synaptic strength in the hippocampus, where the synaptic loss was dominant (i.e., stratum radiatum), we measured both the amplitude of the evoked presynaptic compound action potential (i.e., fiber volley) and of synaptic transmission via measurements of the rising slope of the EPSP in stratum radiatum in CA1 area. From these two measures, we constructed fiber volley versus fEPSP I/O curves to give an index of synaptic function. Plotting one measure against the other, fiber volley versus EPSP, 
A

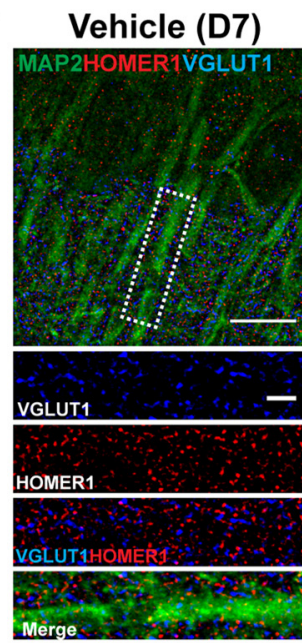

B

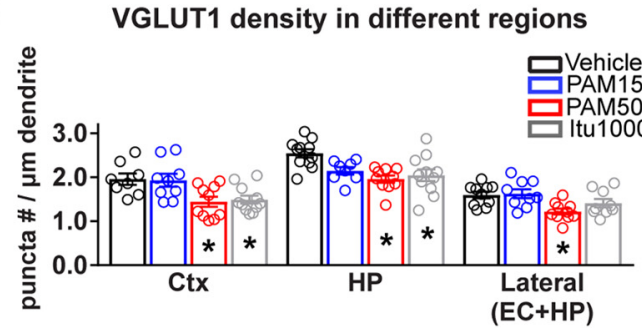

PAM150 (D7)
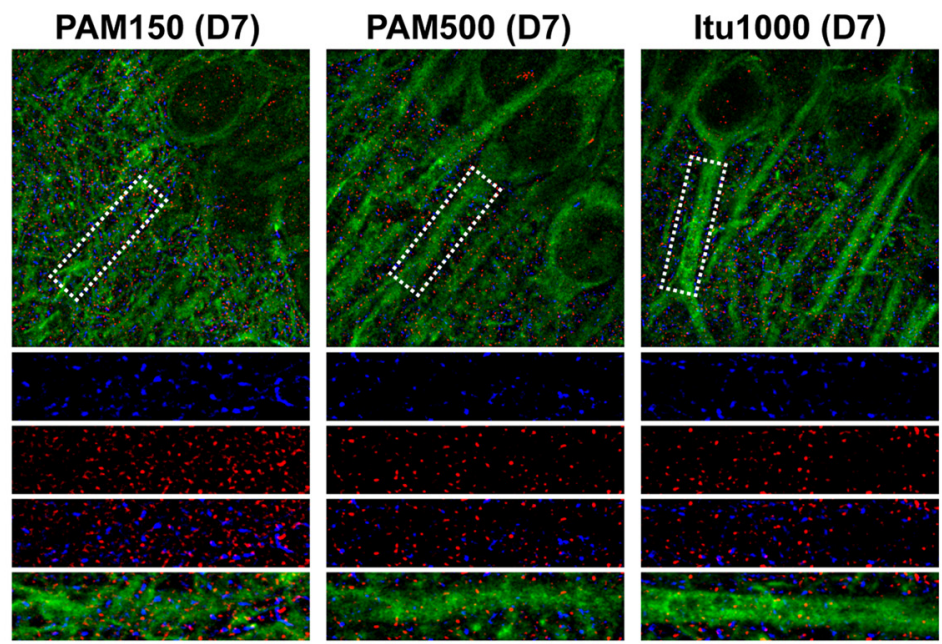

HOMER1 density in different regions

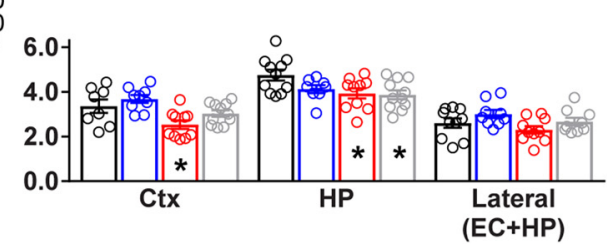

Figure 5. Altered numbers of synaptic puncta by PAM and Itu in brain tissues. A, Fluorescent images of hippocampal regions (stratum radiatum in CA1) stained with anti-MAP2 (green), anti-VGluT1 (blue), and anti-Homer1 (red) at day 7 after injection of vehicle, PAM150, PAM500, and Itu1000 (top from left to right, scale bar, $10 \mu \mathrm{m}$ ). The panels of second, third, fourth, and fifth row show magnified images of a boxed region from top panels in the order of presynapse (VGluT1), postsynapse (Homer1), VGluT1/Homer1, and MAP2/VGluT1/Homer1, respectively (scale bar, 2 $\mu \mathrm{m})$. B, Quantification of VGluT1 and Homer1 puncta in the regions of $\mathrm{Ctx}, \mathrm{HP}$, and EC/HP. The number of VGluT1 puncta in Ctx and HP was significantly decreased by both PAM500 ( $p_{\mathrm{Ctx}}=0.0264$, $p_{\mathrm{HP}}=0.0015$ vs vehicle) and Itu1000 ( $p_{\mathrm{Ctx}}=0.0445, p_{\mathrm{HP}}=0.0066 \mathrm{vs}$ vehicle). The number of VGluT1 puncta in EC/HP was decreased only by PAM500 ( $p=0.014$ vs vehicle). The number of Homer1 puncta in HP was significantly decreased by both PAM500 ( $p_{\mathrm{HP}}=0.0459$ vs vehicle) and Itu1000 ( $p_{\mathrm{HP}}=0.0231$ vs vehicle). The number of Homer1 puncta in Ctx was decreased only by PAM500 ( $p=0.0363$ vs vehicle). The number of Homer1 puncta in EC/HP was not altered by either PAM or Itu. Data are presented as mean of puncta number/dendrite length (in micrometers) \pm SEM of $n=8-11$ fields of views collected from 4 independent experiments. ${ }^{*} p<0.05$.

gives an index of how successfully activated axons in turn activate synapses.

The axonal function did not appear to be disturbed by PAM or Itu because the stimulus versus fiber volley I/O curves were not significantly different among the three conditions $(p=0.78$; Fig. $7 B)$. However, both PAM and Itu caused significant changes in the synaptic transmission resulting from the activation of these axons. The fiber volley versus fEPSP I/O curve (Fig. 7C) showed a significant change in synaptic transmission in response to both PAM500 and Itu1000 challenges. However, these changes in synaptic transmission were not in the expected direction. Rather than a decrease in synaptic efficacy, both PAM and Itu caused no significant change in the left, low-stimulus-strength portion of the curves, but significantly increased the maximum levels at the right, high-stimulus-strength portion of the curve, compared with the vehicle control $(p=0.0015)$. These results suggest a complex response to PAM and Itu in the affected synaptic populations, which could be related to discrepancy of synaptic density between areas within the hippocampus (Figs. 5, 6). Conversely, all synapses we recorded were impaired in one significant function: plasticity as revealed by LTP. LTP was significantly impaired by both PAM500 and Itu1000 ( $p<0.001$ vs vehicle; Fig. 7D).

PAM decreases the number of presynaptic sites in hippocampal neuron cultures

To further investigate how PAM disrupts synaptic density and function, hippocampal neuron cultures of 18 DIV were incu- bated with PAM and Itu for $18 \mathrm{~h}$ at varied concentrations (PAM: 0, 50, 150, 500, and $2500 \mathrm{ng} / \mathrm{ml}$; Itu: $1000 \mathrm{ng} / \mathrm{ml}$ ). VGluT1 and Homer1 as presynaptic and postsynaptic proteins, respectively, were visualized to probe the changes in the distribution of synaptic sites on dendrites following PAM and Itu incubation (Fig. $8 A$ ). For the quantification of synaptic density, the number of synaptic puncta (i.e., VGluT1 or Homer1) per length of randomly selected secondary dendrite was counted for all conditions. Our results indicated that the number of presynaptic sites in neurons with PAM500 ( $p=0.012)$ and PAM $2500(p=0.002)$ was significantly decreased compared with the vehicle group $(0$ $\mathrm{ng} / \mathrm{ml}$ ), whereas the number of postsynaptic sites remained consistent through all concentrations of PAM tested (Fig. $8 B$ ). These results suggest that a bacterial lipopeptide, PAM, disrupts synaptic connections by damaging exclusively presynaptic sites of neurons. However, the density of neither presynaptic nor postsynaptic sites was altered by the treatment of Itu (1000), which is distinct from the results of in vivo tissue experiments.

\section{PAM reduces the frequency of spontaneous excitatory} synaptic transmission

To determine whether the disruption of presynaptic sites by PAM causes a physiological change (i.e., synaptic dysfunction), we conducted whole-cell patch-clamp recording on cultured hippocampal neurons (18 DIV) exposed to different concentrations of PAM $(0,150,500$, and $2500 \mathrm{ng} / \mathrm{ml}$ for $18 \mathrm{~h})$ to measure spontaneous synaptic transmission (mEPSCs) (Fig. 8C,D). The results 
A

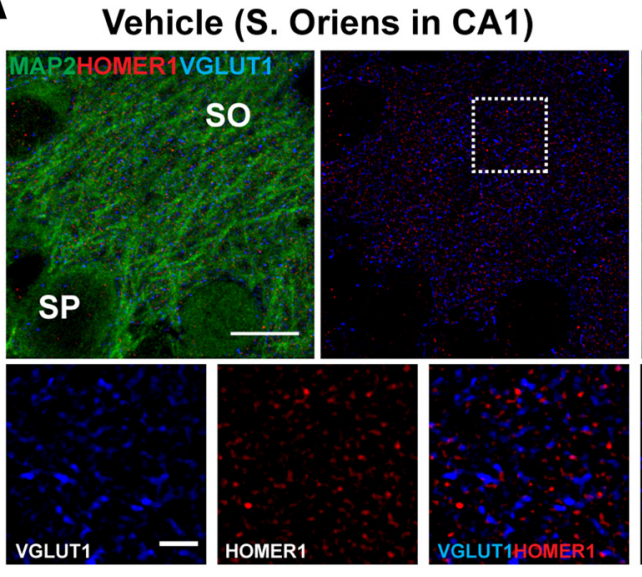

B

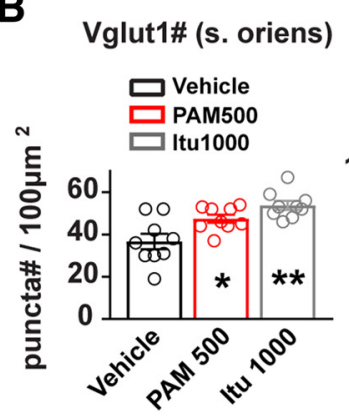

Homer1\# (s. oriens)

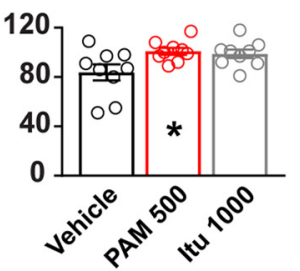

PAM500 (S. Oriens in CA1)

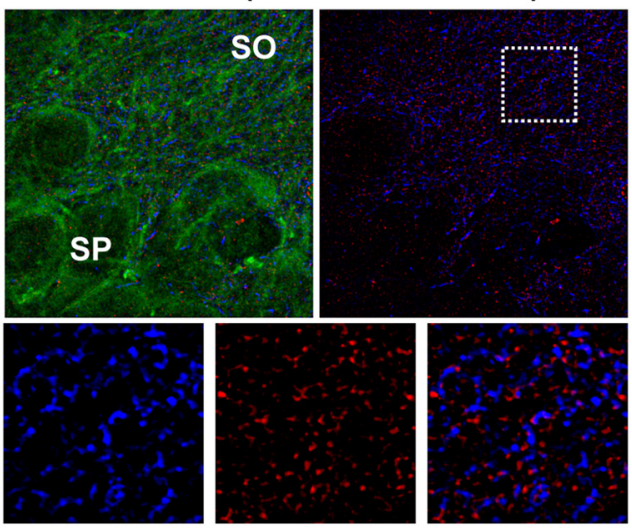

C Injection of PAM/ltu

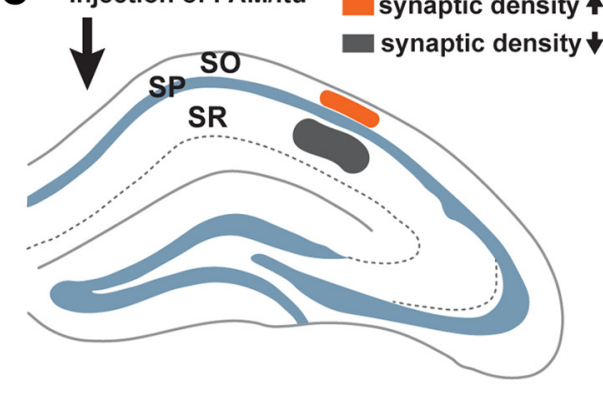

Figure 6. Increased numbers of synaptic puncta by PAM and Itu in stratum oriens in CA1. A, Fluorescent images of stratum oriens in CA1 area stained with anti-MAP2 (green), anti-VGluT1 (blue), and anti-Homer1 (red) at day 7 after injection of vehicle and PAM500 (top, scale bar, $10 \mu \mathrm{m}$ ). SO, Stratum oriens; SP, stratum pyramidale. Bottom shows magnified images of a boxed region from the top in the order of presynapse (VGluT1), postsynapse (Homer1), and VGluT1/Homer1, respectively (scale bar, $2 \mu \mathrm{m}$ ). B, Quantification of VGluT1 and Homer1 puncta in S0. Because it is difficult to trace a dendrite in this region, the number of synaptic puncta was averaged by a defined area (i.e., $\left.100 \mu \mathrm{m}^{2}\right)$ in S0. The number of VGluT1 puncta was significantly increased by both PAM500 ( $p=$ 0.0215 vs vehicle) and Itu 1000 ( $p=0.0003$ vs vehicle). The number of Homer 1 puncta was significantly increased by PAM500 ( $p=0.0343$ vs vehicle). Itu1000 also increased the number of Homer1 puncta, but this did not reach statistical significance ( $p=0.0688$ vs vehicle). Data are presented as mean of puncta number $/ 100 \mu \mathrm{m}^{2} \pm$ SEM of $n=9$ fields of view collected from 3 independent experiments. ${ }^{*} p<0.05,{ }^{* *} p<0.001$, one-way ANOVA. C, Summary scheme of synaptic puncta density in CA1 area. Synaptic density in S0 in CA1 was significantly increased, whereas synaptic density in SR in CA1 was significantly decreased (Fig. 5).

showed that the increase of PAM concentrations shifted the cumulative probability distribution significantly toward higher interevent intervals (i.e., decreased frequency) $\left(p_{\text {PAM500, PAM2500 }}<\right.$ 0.0001 vs vehicle, K-S test) (Fig. $8 E$ ) and also significantly decreased the mean mEPSC frequency $\left(p_{\text {PAM500 }}=0.037, p_{\text {PAM2500 }}\right.$ $<0.001$ vs vehicle, one-way ANOVA) (Fig. $8 F$ ), which indicates the alteration of presynaptic strength. In contrast, a cumulative distribution of mEPSC amplitudes did not show a clear shift toward any direction by treatment of PAM and Itu even though the statistics showed an altered cumulative distribution compared with vehicle $\left(p_{\text {PAM } 150}=0.0018, p_{\text {PAM500 }}<0.0001\right.$, $p_{\text {PAM } 2500}=0.0029, p_{\text {Itu } 1000}=0.0004$ vs vehicle, K-S test) (Fig. $8 E)$. The group comparison also showed that the mean mEPSC amplitude did not show significant difference among the groups ( $p>0.05$ vs vehicle, one-way ANOVA) (Fig. $8 F$ ), which indicates no significant postsynaptic responsiveness. These results suggest that a higher concentration of PAM disrupts synaptic transmission possibly by damaging presynaptic sites. We also examined the effect of Itu1000 on the mean frequency and amplitude of mEPSCs and found that the vehicle and Itu showed a similar effect in neuron cultures (Fig. $8 F$ ). Therefore, we confirmed that the decreased synaptic transmission of cultured neurons by PAM (500 and 2500) is consistent with our result above showing the decreased number of presynaptic puncta in cultured neurons by PAM (500 and 2500).
PAM induces aggregation of synapses and does not alter the expression level of synaptic proteins

The high concentrations of PAM (500 and $2500 \mathrm{ng} / \mathrm{ml}$ ) induced a number of aggregated forms of synapses (VGluT1 and Homer1) around dendrites (Fig. 8G). To determine whether the expression level of synaptic proteins is altered by the synaptic aggregation, we performed Western blot assay for VGluT1 and Homer1 under varied concentrations of PAM and Itu (Fig. $8 H, I$ ). The densitometry analysis revealed that the expression level of VGluT1 was decreased only by PAM2500 ( $p=0.015)$, whereas the expression level of Homer1 was not altered by any of groups tested, which is similar to the results from in vivo experiments.

\section{PAM decreases the dendritic area in Ctx, HP, and EC/HP}

To investigate whether PAM and Itu induce a dendritic alteration in brain tissues, we measured the fluorescent intensity and area coverage of MAP2 in Ctx, HP, and EC/HP (Fig. 9B). Compared with vehicle, PAM500 significantly decreased signal intensity of MAP2 in Ctx $(p=0.009), \mathrm{HP}(p=0.0452)$, and EC/HP $(p=$ $0.048)$ and also decreased area coverage of MAP 2 in EC/HP $(p=$ $0.043)$, whereas Itu1000 decreased signal intensity $(p=0.003)$ and area coverage $(p=0.017)$ of MAP2 only in Ctx. With respect to changes in terms of time courses from day 1 to day 7 after injection, both signal intensity and area coverage of MAP2 were aggravated by PAM500 in the regions of HP ( $p_{\text {Intensity D1 vs D7 }}=$ 
A

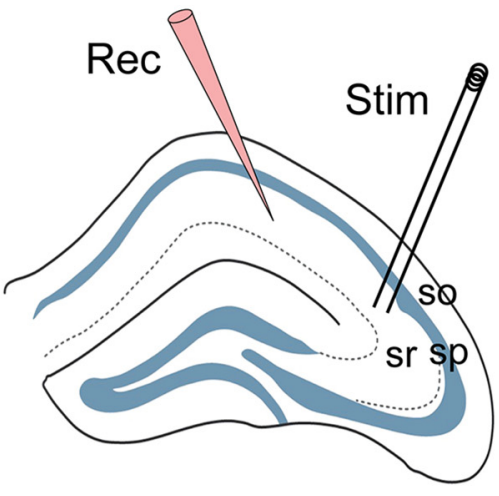

\section{C}

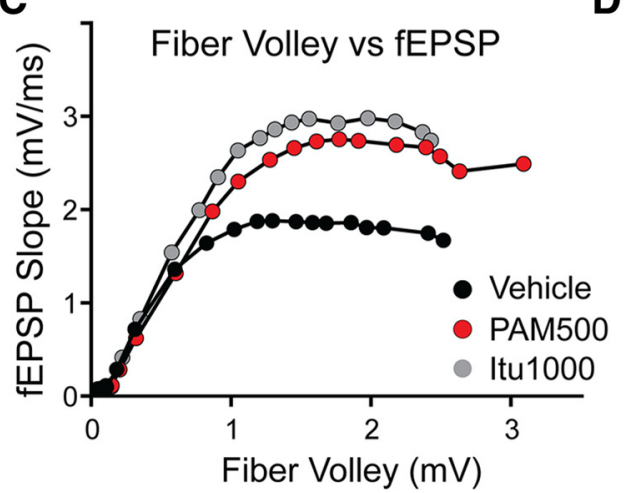

B
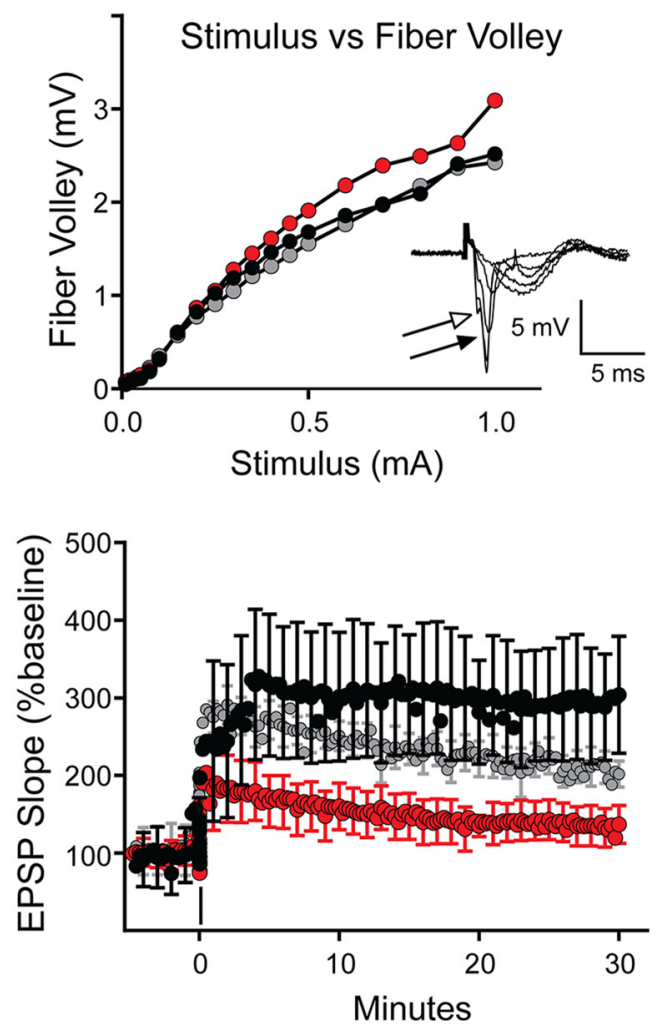

Figure 7. I/ 0 curves and LTP in PAM500/Itu1000-treated mouse hippocampus. A, Schematic diagram of a hippocampal slice showing the electrode placement for these experiments. A concentric bipolar stimulating electrode (Stim) was placed in the stratum radiatum (sr) near the CA1/CA2 border and a saline-filled glass electrode (Rec) was placed in the stratum radiatum $\sim 2 / 3$ the distance from CA2 to subiculum. so, Stratum oriens; sp, stratum pyramidale; sr, stratum radiatum. $B$, Average relationship between the stimulus and the fiber volley evoked by a range of stimulus strengths. Inset, Exemplar field potentials at five different stimulus strengths. Open arrow: fiber volley; closed arrow, EPSP rising slope. C, Relationship between the amplitude of the fiber volley and the EPSP rising slope. Shown is the average of eight experiments in vehicle and nine in PAM500 and Itu1000. Error bars have been omitted for visual clarity. The curves in $B$ are not significantly different from one another ( $p>0.98, \mathrm{~K}-\mathrm{S})$, whereas in C, both PAM500 and Itu 1000 are significantly larger than vehicle ( $p=0.015$ vs vehicle); PAM500 and Itu 1000 are not significantly different from each other, $p=0.082)$. D, LTP induced by theta-burst stimulus (arrow) in vehicle-treated slices (295\% $\pm 75.7 \%)$, PAM500-treated slices (135.4 $\pm 24.9 \%)$, and Itu1000-treated slices (207.1 $\pm 22.1 \%$; averages of last $5 \mathrm{~min} \pm \mathrm{SEM}$; all significantly different from one-another at $p<0.001$ by ANOVA for repeated measures).

$\left.0.007, p_{\text {Area D1 vs D7 }}=0.012\right)$, and EC/HP $\left(p_{\text {Intensity D1 vs D7 }}=\right.$ $0.004, p_{\text {Area D1 vs D7 }}=0.018$ ) over time.

Dendrite branching is altered by a high concentration of PAM To further investigate dendritic morphology of neurons in response to PAM and Itu, dendrite branching of cultured neurons stained with MAP2 was analyzed with respect to the number of primary dendrites (Fig. 10B), the extension range of apical dendrites (Fig. 10C), the number of branch nodes/apical dendrite (Fig. 10D), and the number of branch nodes/basal dendrite (Fig. $10 E)$. None of these parameters was altered by vehicle, PAM150, PAM500, PAM2500, or Itu1000 with statistical significance. To determine the number of intersections at different extension range of dendrites, manually traced dendrites were superimposed on circle masks ranging from 20 to $200 \mu \mathrm{m}$. The result showed that PAM2500 significantly decreased the number of intersections of apical dendrites at the range of $60(p=0.011), 80(p=$ $0.035)$, and $100 \mu \mathrm{m}(p=0.046)$ compared with vehicle (Fig. $10 F)$. However, the number of intersections of basal dendrites was not altered at any range of dendrite extension by any condition (Fig. 10G).

PAM and Itu do not induce apoptosis of neurons in either in vivo tissues or in vitro cultures

Both in vivo and in vitro experiments revealed that PAM damaged synapses and dendrites. To determine whether the synaptic and dendritic damages lead to the death of neurons, we performed TUNEL assay of brain tissues and neuron cultures under varied concentrations of PAM and Itu. Unexpectedly, PAM did not induce apoptosis of neurons in either brain tissues or in vitro cultures (Fig. 11). In the regions of Ctx, HP, and EC/HP where PAM or Itu aggravated synaptic function and network, the ratio of TUNEL-positive neurons was extremely low $(<5 \%)$ throughout all conditions (vehicle, PAM150, PAM500, and Itu1000) (Fig. 11B). Only the region of injection path in Ctx showed a higher number of TUNEL-positive cells regardless of injection molecules including vehicle (water) (Fig. 11C). At day 7 after injection, the ratio of TUNELpositive neurons in the injection area was significantly higher than those in other brain regions (Fig. 11D). TUNEL assay of in vitro cultures also showed that none of high concentrations of PAM or Itu induced apoptosis of neurons (Fig. 11E,F).

\section{Discussion}

The role of neuronal activity in neuroinflammation is emerging because conventional neuroinflammation studies with an emphasis on the inflammatory activity of microglia and astrocytes have provided incomplete understanding of functional abnormalities in the course of brain infection (Xanthos and Sandkühler, 2014). Therefore, we investigated the effect of bacterial lipopeptides on neuron-specific dysfunctions by performing functional brain imaging, in vivo brain tissue studies, and in vitro neuron culture studies in parallel. For functional brain imaging, 


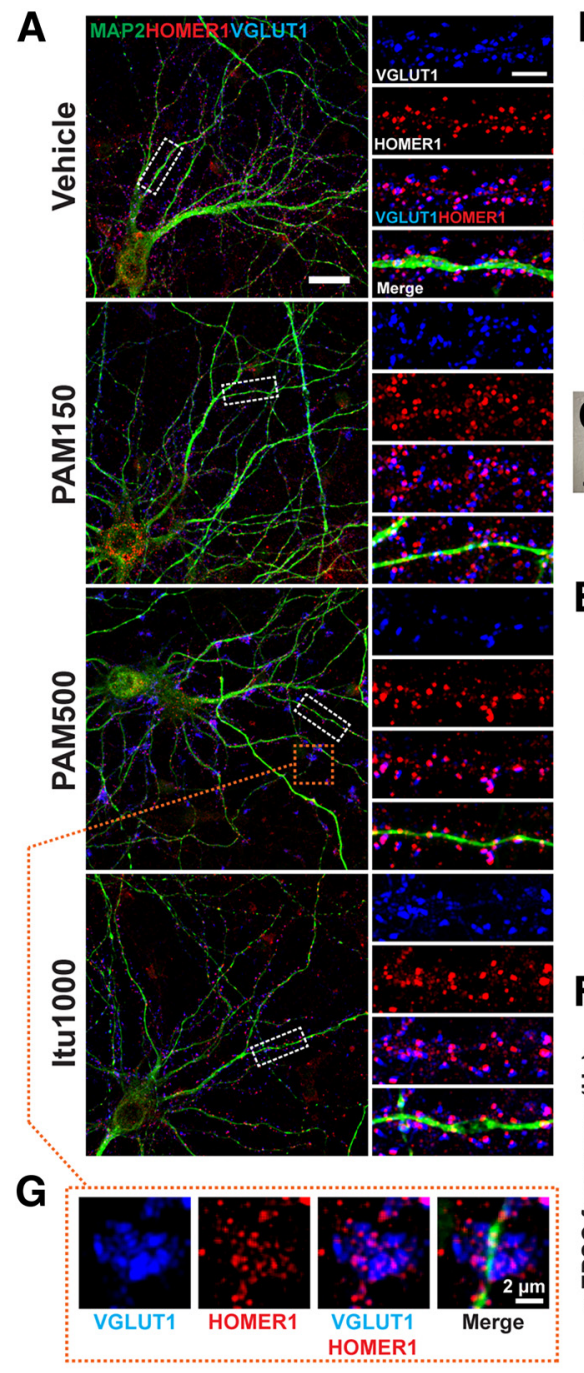

B VGLUT1 (presynapse) HOMER1 (postsynapse)

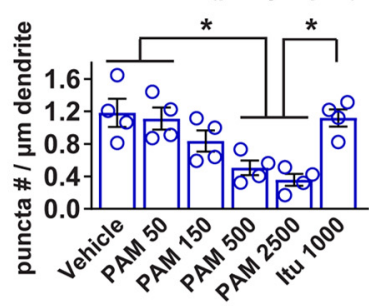

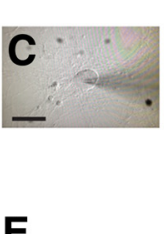

$\mathbf{E}$
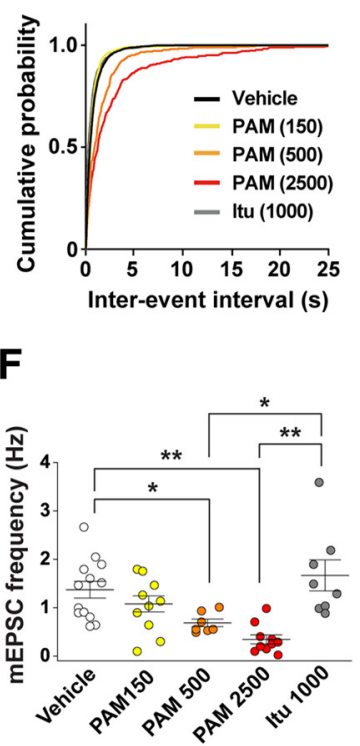

1

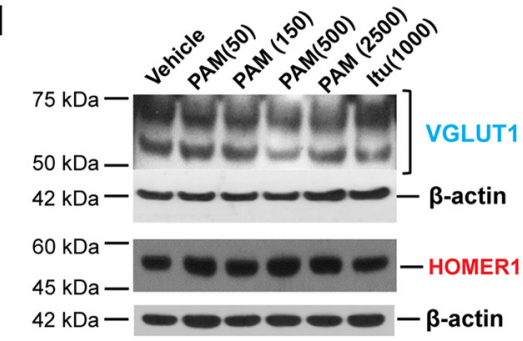

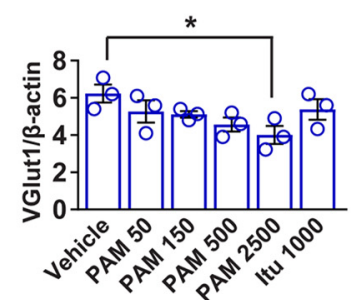
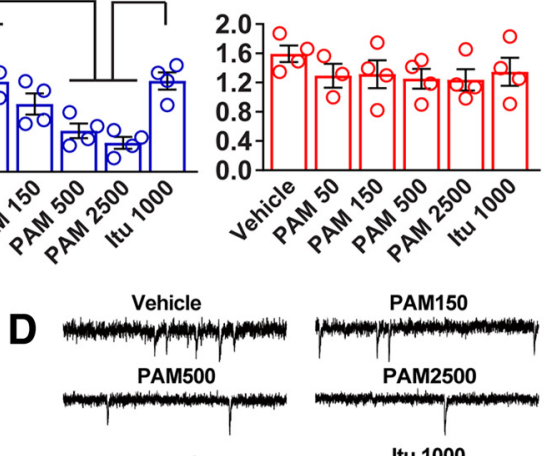

Itu 1000 $\frac{1}{100 \mathrm{~ms}} \mathrm{pA}$
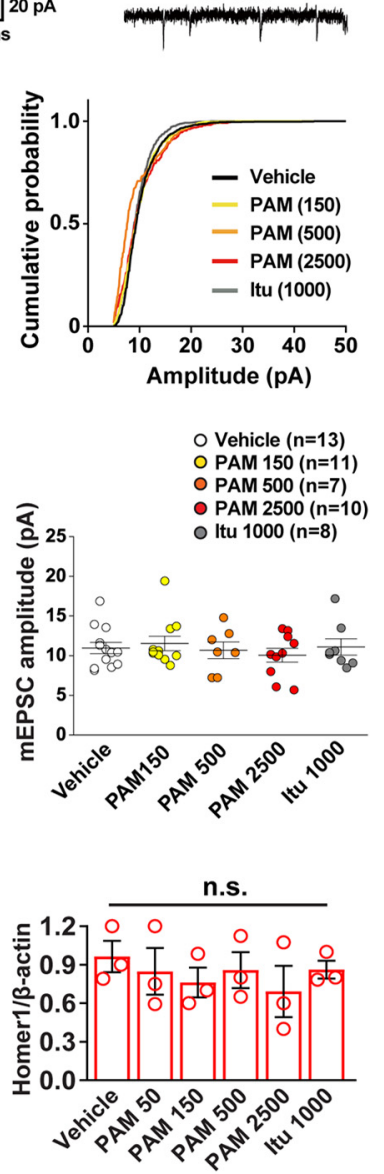

Figure 8. Effect of PAM on synaptic alteration in cultured neurons. Embryonic day 18 rat hippocampal neurons were incubated with vehicle (water) or with PAM50, PAM150, PAM500, or PAM2500 for $18 \mathrm{~h}$. Itu1000 was used as a negative control for PAM. A, Left, Fluorescent images showing presynapses (VGluT1, Blue), postsynapses (Homer1, Red), and dendrites (MAP2, green). Scale bar, $20 \mu \mathrm{m}$. Right, Magnified images of dendrites (MAP2) costained with VGluT1 and Homer1. Scale bar, $5 \mu \mathrm{m}$. B, Number of VGlutT1 puncta was significantly decreased by PAM500 and PAM2500 compared with those by vehicle, PAM50, and Itu1000. The number of Homer 1 puncta remained consistent through different concentrations of PAM and Itu. Data are presented as mean of puncta number/dendrite length (in micrometers) \pm SEM of $n=3-4$ independent experiments. ${ }^{*} p<0.05$. C $-\boldsymbol{F}$, Spontaneous excitatory synaptic transmission (mEPSCS) of cultured hippocampal neurons with PAM and Itu. C, Representative IR-DIC image showing whole-cell patch-clamp recording. Scale bar, $20 \mu \mathrm{m}$. D, Sample recording traces for mEPSC with PAM and Itu. E, Cumulative probability distributions of all $\mathrm{mEPSC}$ interevent intervals and all amplitudes (cells pooled). PAM and Itu significantly altered both the interevent interval ( $p_{\text {PAM500, PAM2500, It } 1000}<0.0001$ vs vehicle, $K-S$ test) and amplitude ( $p_{\text {PAM500, PAM2500, Itu1000 }}<0.05$ vs vehicle, K-S test). $F$, Mean frequency and amplitude of mEPSCs (per each cell). The frequency of mEPSCS with PAM (500 and 2500$)$ was significantly lower than that with the vehicle or $\operatorname{Itu}\left(F_{(4,44)}=7.813,{ }^{*} p<0.05,{ }^{* *} p<0.001\right.$, one-way ANOVA). The amplitude of mEPS(s was not altered by any treatment group $\left(F_{(4,44)}=0.397, p>0.05\right.$, one-way ANOVA). G, Representative image showing synaptic aggregation at higher concentrations of PAM500 and PAM2500. VGluT1 and Homer1 pucnta were aggregated around dendrites. Scale bar, $2 \mu \mathrm{m}$. $\boldsymbol{H}$, Representative immunoblots of VGluT1 and Homer1 from varied concentrations of PAM and Itu. I, Protein band densitometry showing that the expression of VGluT1 was not decreased until the concentration of PAM increased up to $2500 \mathrm{ng} / \mathrm{ml}$. ${ }^{*} p<0.05$. The expression level of Homer 1 was not altered by PAM or Itu.

we used $\left[{ }^{18} \mathrm{~F}\right]$ FMZ PET to gain information more specific to the density of neurons with active neurotransmitter binding (i.e., neuronal integrity) in the brain. Our analysis revealed that the abnormal metabolic activity induced by PAM and Itu was found mainly in the injection area (i.e., hippocampus) and recovered within 1 week, whereas the altered neuronal integrity induced by PAM and Itu only partially recovered. The abnormal neuronal integrity in bilateral hippocampus and EC still remained after 1 week (Fig. 1B). A circuit from EC to CA1 has been considered a region with strong presynaptic inputs (Oh et al., 2014), which 
A Vehicle (D7)

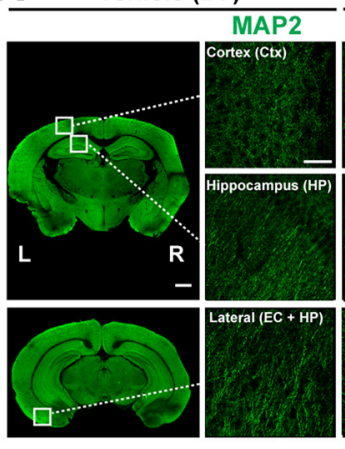

PAM500 (D7)

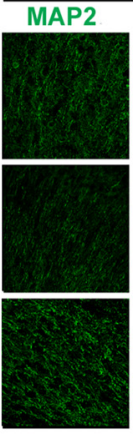

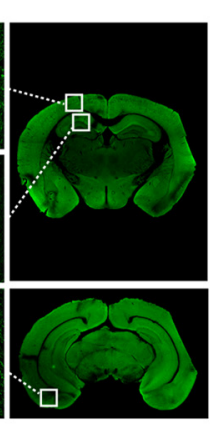
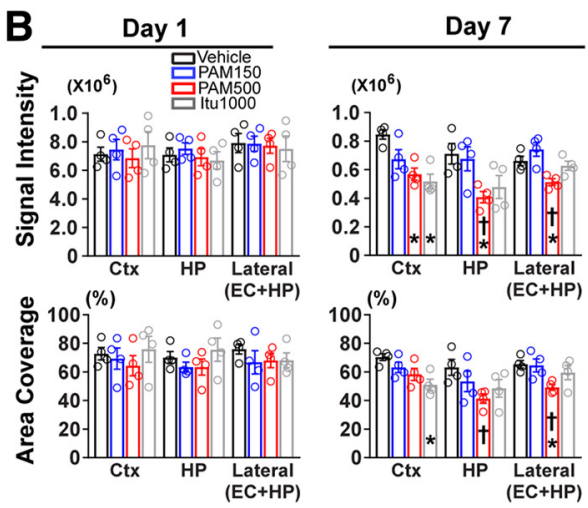

Figure 9. Effect of PAM on dendriticalteration in brain tissues. $A$, Fluorescent images of brain sections stained with MAP2 (green) at day 7 after injection of vehicle (left) and PAM500 (right). Boxes in the image of whole-brain section (scale bar, $500 \mu \mathrm{m}$ ) indicate the regions (Ctx, HP, and lateral EC, scale bar, $50 \mu \mathrm{m}$ ) where the integrated density of fluorescent intensity from dendrites (MAP2) was measured (see Materials and Methods). B, Quantification of the integrated density of fluorescent intensity from MAP2 at day 1 and day 7 after injection. Compared with vehicle, PAM500 significantly decreased the signal intensity of MAP2 in all regions (Ctx, HP, and EC) tested at day 7 after injection ( ${ }^{*} p<0.05$ vs vehicle). Compared with analysis at day 1, PAM500 significantly decreased the signal intensity of MAP2 in both HP and EC region ( $t p<0.05$ vs PAM500 ${ }_{\text {Day } 1 \text {-HPI }}$, PAM500 Day 1-Lateral $)$. All data are presented as mean \pm SEM of $n=4$ independent experiments. The MAP2 area coverage in the region of $\mathrm{Ctx}, \mathrm{HP}$, and lateral (EC $+\mathrm{HP})$ was measured. Compared with vehicle, PAM500 significantly decreased the MAP2 area coverage in EC, wheresa Itu1000 significantly decreased the MAP2 area coverage in Ctx. Compared with analysis at day 1, PAM500 significantly decreased the signal intensity of MAP2 in both HP and EC region ( $† p<0.05$ vs PAM500 Day 1-HP, PAM500 Day 1-Lateral $)$. All data are presented as mean \pm SEM of $n=4$ independent experiments.
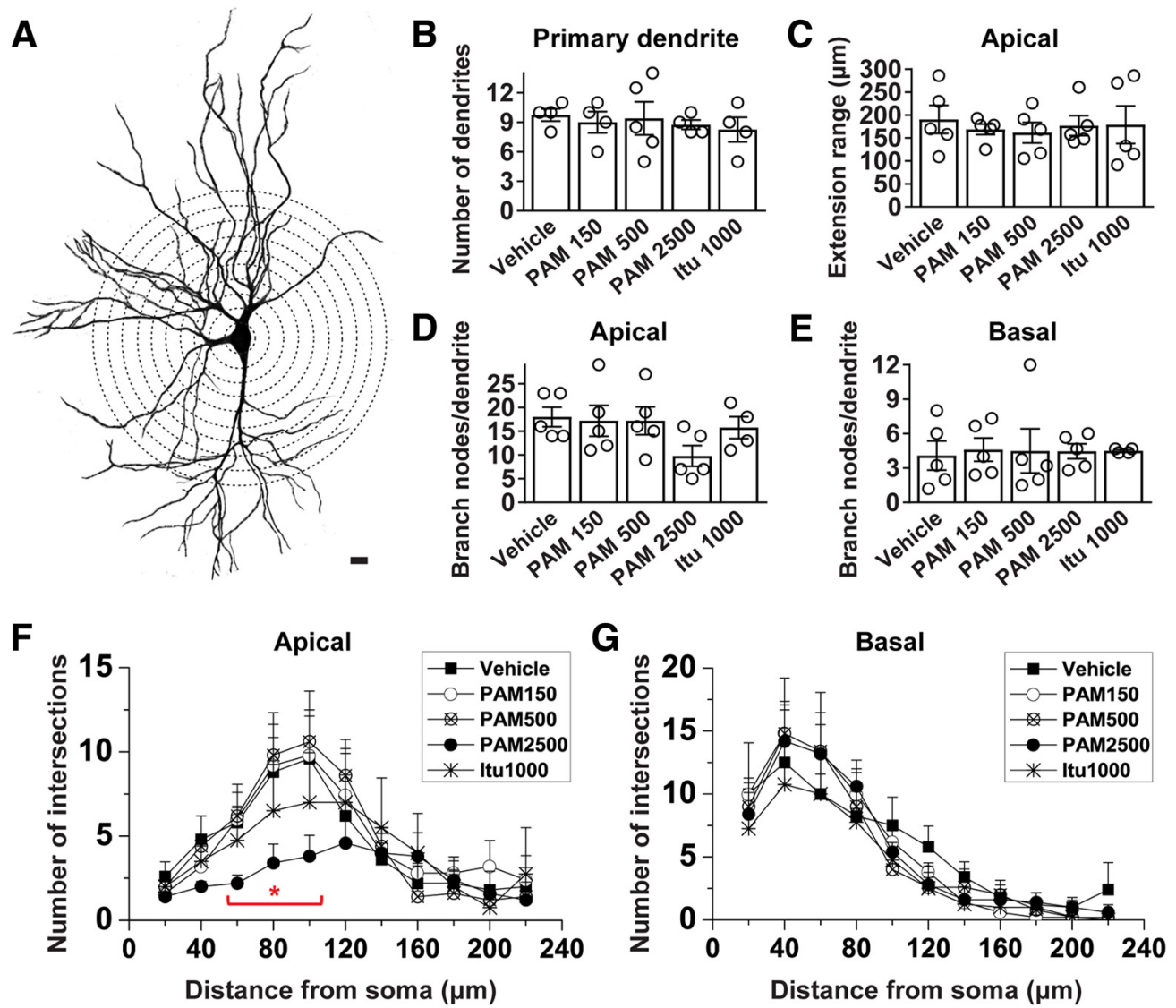

Figure 10. Analysis of branching morphology dendrites in response to PAM and Itu treatment in vitro. A, Manual drawing of dendrites in MAP2-positive neuron in vitro. Template circles ranging from 20 to $200 \mu \mathrm{m}$ were superimposed. Scale bar, $20 \mu \mathrm{m}$. Following $18 \mathrm{~h}$ treatment of PAM150, PAM500, PAM2500, and Itu1000 in embryonic day 18 rat hippocampal cultures, dendritic morphology was analyzed in terms of the number of primary dendrites $(\boldsymbol{B})$, the extension range of apical dendrites $(\boldsymbol{C})$, the number of branch nodes/apical dendrites $(\boldsymbol{D})$, and the number of branch nodes/basal dendrite $(\boldsymbol{E}) . \boldsymbol{F}$, Number of intersections of apical dendrites measured on template circles. PAM2500 significantly decreased the number of intersections in the range of 60 to $100 \mu \mathrm{m}$ compared with vehicle $\left.{ }^{*} p<0.05\right)$. G, Number of intersections of basal dendrites was measured and the result showed no significant differences throughout all conditions. All data are presented as mean \pm SEM of $n=4$ to 5 independent experiments.

could be associated with our in vivo and in vitro studies where PAM damaged exclusively presynaptic sites (Figs. 4, 5).

Using PET data, we also constructed metabolic and neuronal networks to determine altered correlation between various regions in the brain injected with PAM and Itu. FDG PET imaging showed that the abnormal metabolic network induced by PAM500 and Itu1000 recovered in 1 week (Fig. 3A), which was similar to the metabolic activity profile (Fig. $1 A$ ). 
A
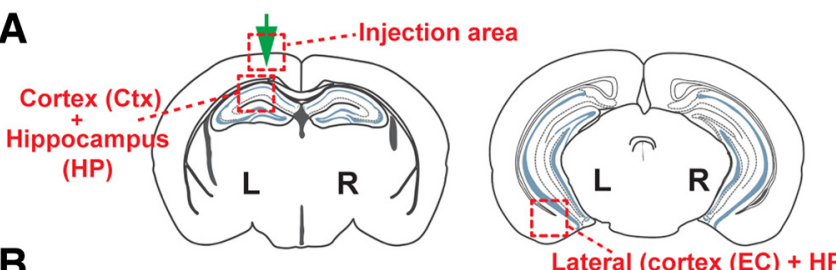

C

Lateral (cortex (EC) + HP)

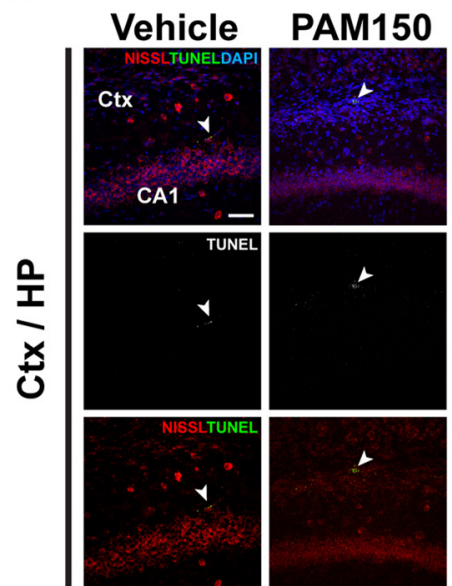

PAM500

Itu1000
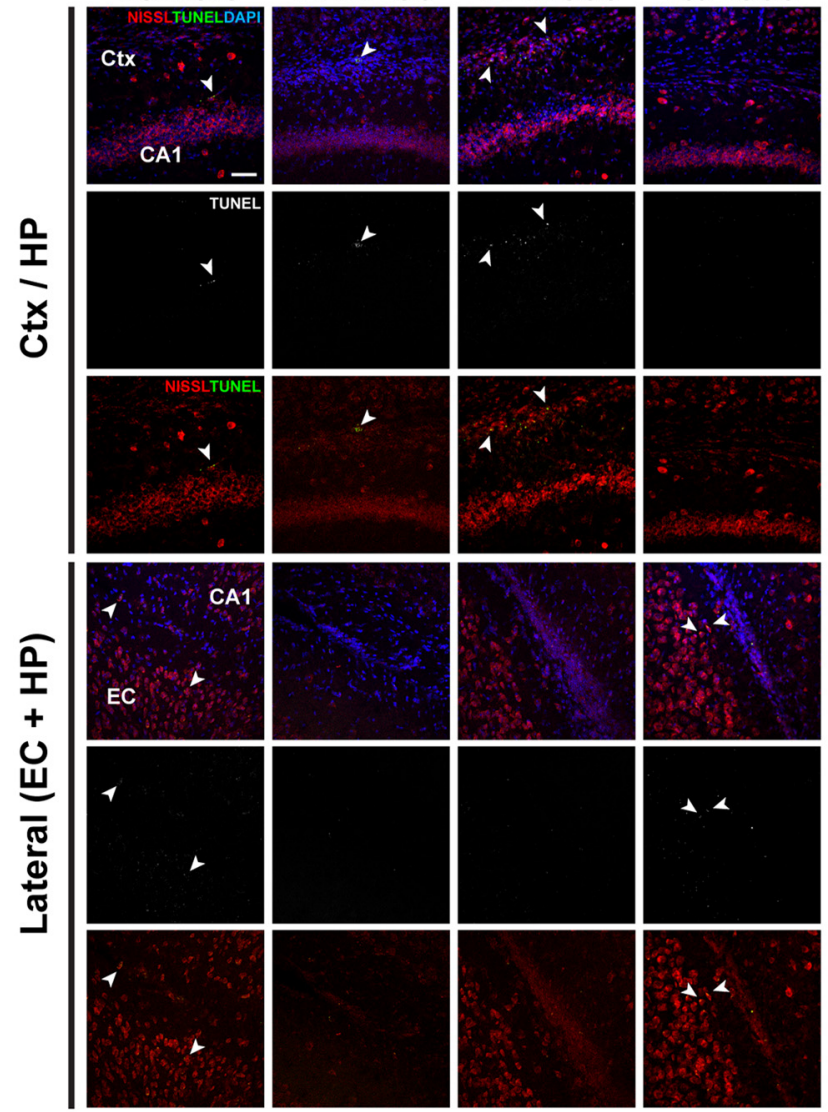

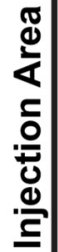

Vehicle PAM500

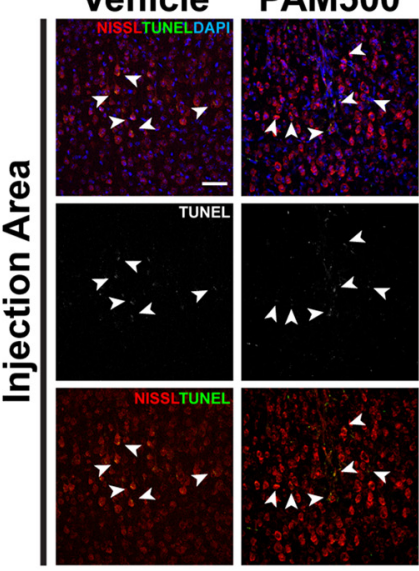

D
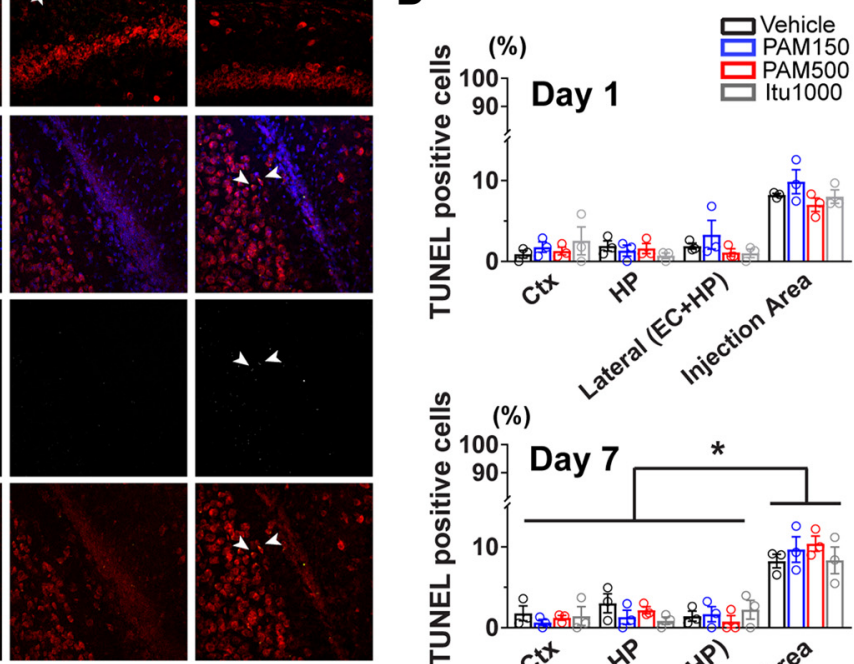

E
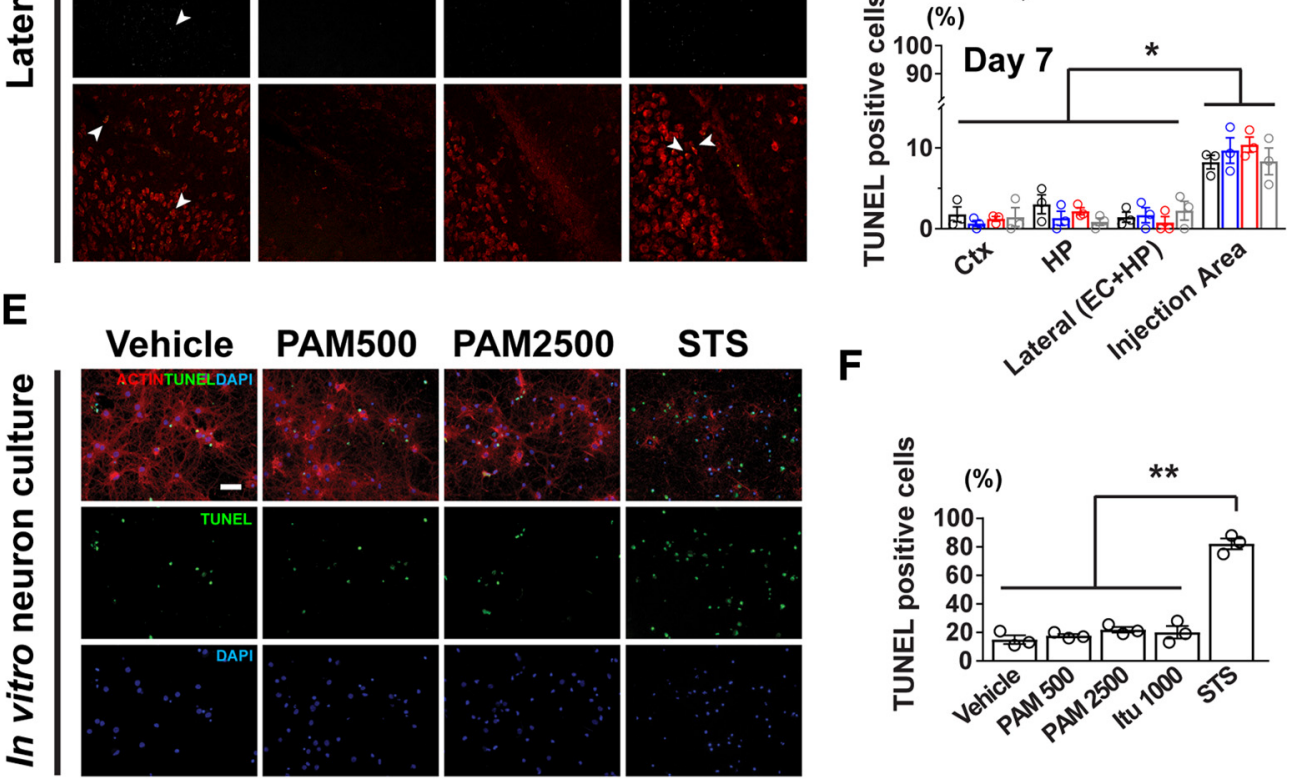

$\mathbf{F}$
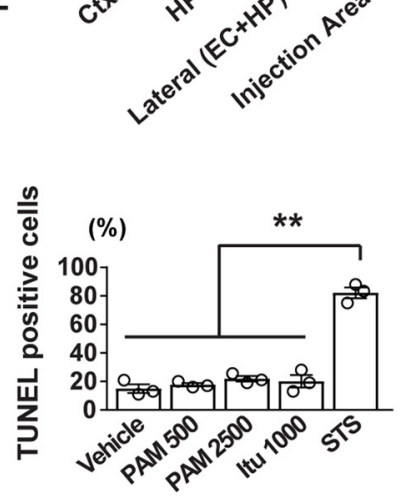

Figure 11. In vivo and in vitro TUNEL assay following PAM and Itu treatment. $A$, Scheme of brain section showing three regions (Ctx/HP, EC, and injection area) where TUNEL assay was performed. $\boldsymbol{B}$, TUNEL images of Ctx/HP and EC at day 7 after PAM and Itu injections. C, TUNEL images of injection area at day 7 after PAM and Itu injections. $\boldsymbol{D}$, Ratio of TUNEL-positive signal at day 1 and day 7 after injection. Only the TUNEL signal colocalized with NissI (red) and DAPI (blue) was counted. There was no statistical difference among regions at day 1 ( $p>0.05$ ). At day 7 after injection, the level of TUNEL signal in the injection area was significantly higher than other regions regardless of condition groups $\left({ }^{*} p<0.05\right)$. Data are presented as mean \pm SEM of $n=3$ independent experiments. $E$, Representative TUNEL images of embryonic day 18 rat hippocampal neuron cultures incubated with vehicle (water), PAM500, PAM2500, and STS $(0.75 \mu \mathrm{m})$ for $18 \mathrm{~h}$. F, Quantitative evaluation of TUNEL-positive cells. Only the TUNEL signal colocalized with actin (red) and DAPI (blue) was counted. STS was used to induce a positive TUNEL signal in cultures. High concentrations of PAM (500 and 2500) and Itu1000 did not induce apoptosis of cultured neurons. ${ }^{* *} p<0.001$ vs STS. Error bars indicate mean \pm SEM of $n=3$ independent experiments. Scale bar, $50 \mu \mathrm{m}$. 
The recovery of metabolic activity and network could possibly be related to the result showing that PAM did not induce neuronal apoptosis (Fig. 11). The attenuation of inflammatory activities of microglia or astrocytes might also have contributed to the recovery of metabolic activity and network (Buscombe and Signore, 2003).

In contrast, the FMZ PET imaging revealed that the abnormal neuronal network induced by PAM and Itu persisted for 1 week, which involved amygdala, fornix, hippocampus, olfactory bulb, and thalamus. This suggests that the bacterial lipopeptides injected into CA1 disrupt neuronal networks exclusively in the path of the limbic system (Fig. 3B, Table 4). Compared with Itu, PAM involved more VOIs with dysfunctional networks, which were mainly located on the side of injections (i.e., left hemisphere). Considering the difference of concentrations between PAM (500 $\mathrm{ng} / \mathrm{ml}, 0.33 \mu \mathrm{M})$ and Itu $(1000 \mathrm{ng} / \mathrm{ml}, 1 \mu \mathrm{M})$, the effect of PAM on functional abnormalities in the brain is more aggressive and localized than that of Itu, which showed milder, nonspecific, and wider damage in the brain.

To investigate whether the abnormal neuronal activity and network induced by PAM and Itu is correlated to synaptic impairment, we measured the density and function of synapses in the brain tissues injected with PAM and Itu. Our results confirmed that the regions (i.e., HP, lateral EC/HP) where PAM500 induced a reduction of $\mathrm{FMZ} \mathrm{BP}_{\mathrm{ND}}$ interregional correlation (Fig. $3 B$ ) showed a decreased level of synaptic density (Figs. $4 B, 5 B$ ) and dendritic density (Fig. 9B).

In the study of synaptic function, we found that the fiber volley I/O was not affected by treatment with PAM500 or Itu1000. This suggests that there was no detectable reorganization of the axonal network within hippocampal slices. However, the synaptic response driven by those axons was significantly increased by PAM or Itu treatment. This is not the result that we, a priori, would have expected from the I/O measurement. Generally, we expected that a decrease in the anatomical density of synapses would be reflected in a decrease in the amplitude of the I/O curve, not the apparent increase that we did observe. We think that there are two potential explanations for this seeming mismatch between the decrease in the physical synaptic density in the stratum radiatum and the apparent increase in synaptic function reflected in the I/O. The first of these potential explanations is that, whereas the number of synapses was decreased, the strength of the remaining synapses was increased. Consistent with this idea, we found that LTP was decreased in the PAM conditions. If PAM treatment somehow caused a potentiation of these remaining synapses, then LTP evoked after treatment would appear smaller because LTP is saturable (Barnes et al., 1994). The second potential explanation is that, although synaptic density in locations nearby the recording electrode was decreased, the density may have been increased at more distant anatomical locations. Therefore, the apparent increase in synaptic function might be coming from greater synapse density in stratum oriens. This would make the increase more prominent in the stronger stimulus portions of the I/O curve, as we observed. The main consequence of increasing the stimulus during the production of the I/O curve is to recruit additional axons to fire at increasing distance from the stimulating electrode. Consistent with this idea is the finding that synaptic density in the more distant stratum oriens is increased by PAM treatment (Fig. 6). It should be noted that, contrary to the strictest interpretation of the laminar hypothesis (Andersen et al., 1971), the Schaffer collaterals, which are the presynaptic axons stimulated in these experiments, project to both stratum radiatum and stratum oriens
(Amaral and Witter, 1989). Nonetheless, we consider the first explanation, fewer but more potent synapses, to be more likely, since an increase in synaptic density in stratum oriens would likely show up as an upward current source in the stratum radiatum-recorded field potential.

Interestingly, the level of altered postsynaptic density was not always comparable to that of presynapses. The loss of presynaptic density was more dominant than that of postsynapses in many regions in the brain (Figs. 4, 5). To further investigate whether PAM impaired presynapse and postsynapse separately, we performed in vitro neuron culture studies to measure synaptic density and transmission under varied concentrations of PAM. Our results demonstrated that PAM $>500 \mathrm{ng} / \mathrm{ml}$ induced presynaptic damage, which was determined by the decrease of presynaptic sites (i.e., VGluT1) and mEPSC frequency (Fig. 8). This result may be related to a recent study demonstrating that viral infection by West Nile virus reduced presynaptic density with no change in the number of postsynaptic terminals (Vasek et al., 2016). We believe that this is an important result first showing the effect of bacterial molecules on presynapses and postsynapses separately. The results from synaptic density and function assays suggest that the disrupted neuronal integrity and network shown in PET analysis is closely related to presynaptic alterations by PAM.

The expression level of presynapses in the region of HP was not altered by any of varied concentrations of PAM (Fig. 4E). The expression level of presynapses in cultured neurons was also not altered until PAM reached a high concentration $(2500 \mathrm{ng} / \mathrm{ml})$ (Fig. $8 H$ ). This suggests that the expression level of synaptic proteins is not directly linked to the state of synaptic activity. The discrepancy might be attributed to aggregated synaptic proteins caused by PAM (500 and 2500) (Fig. 5G) because these aggregated proteins could still be detected in Western blot assay even after loss of synaptic functions.

Neither PAM nor Itu induced apoptosis of neurons in either in vivo or in vitro experiments. The injection process seemed to induce apoptosis of neurons in the path of injection area rather than PAM or Itu (Fig. 11D). This result could be associated with FDG PET analysis showing recovery of metabolic activity and network in the brain over time (Figs. $1 A, 3 A$ ).

Our findings have several limitations to be addressed. For functional brain imaging, we measured $\mathrm{BP}_{\mathrm{ND}}$ of $\mathrm{FMZ}$ to GABAergic receptors for analyzing neuronal integrity and networks because most interneurons that connect sensory and/or motor neurons in the brain are GABAergic (Markram et al., 2004). Because the majority of neurons in the CNS are glutamatergic, they are interactive with GABAergic neurons (Kehoe and Vulfius, 2000; Carlsson et al., 2001; Mora et al., 2008); however, further investigation using PET radioligands that probe activities of glutamatergic neurons would need to be considered to provide complementary information to our findings. PET imaging of the metabotropic glutamate receptor subtype 5 (mGluR5) using 3-(6-Methyl-pyridin-2-ylethynyl)-cyclohex-2-enone-O- ${ }^{11} \mathrm{C}$ methyl-oxime $\left(\left[{ }^{11} \mathrm{C}\right] \mathrm{ABP} 688\right)$ (Ametamey et al., 2007) could be used to test this. PET imaging using 1-[2-chlorophenyl]- $N$ methyl- $N$-[1-methyl-propyl]-3-isoquinoline carboxamide $\left(\left[{ }^{11} \mathrm{C}\right]\right.$ PK11195) that binds peripheral benzodiazepine receptors expressed on activated microglia (Banati et al., 2000) could also be taken into consideration to investigate the correlation between immune response and synaptic dysfunction. In addition, our findings need to be further evaluated with human subjects suffering from brain infection such as Lyme neuroborreliosis. These 
would be the steps to take for translating our study into the clinical setting and will be the subjects of our future studies.

In conclusion, the present study has demonstrated characteristic neuronal dysfunction induced by bacterial lipopeptides with multiscale assays ranging from synaptic protein to brain network. Our findings did not provide direct evidence of genetic mechanism via which PAM disrupted synaptic functions. However, our results from in vivo and in vitro studies demonstrated altered function of neural circuitry and loss of presynapses by PAM, which could be further studied for finding therapeutic targets. More importantly, the results from the functional brain imaging using FMZ PET showing dysfunctional activity and network patterns in PAM-injected brains could be potentially applied to diagnosis and monitoring of brains infected with bacteria expressing PAM-containing lipoproteins (e.g., ospA from species of Borrelia).

\section{References}

Akira S, Takeda K (2004) Toll-like receptor signalling. Nat Rev Immunol 4:499-511. CrossRef Medline

Aliprantis AO, Yang RB, Mark MR, Suggett S, Devaux B, Radolf JD, Klimpel GR, Godowski P, Zychlinsky A (1999) Cell activation and apoptosis by bacterial lipoproteins through toll-like receptor-2. Science 285:736-739. CrossRef Medline

Amaral DG, Witter MP (1989) The three-dimensional organization of the hippocampal formation: a review of anatomical data. Neuroscience 31: 571-591. CrossRef Medline

Ametamey SM, Treyer V, Streffer J, Wyss MT, Schmidt M, Blagoev M, Hintermann S, Auberson Y, Gasparini F, Fischer UC, Buck A (2007) Human PET studies of metabotropic glutamate receptor subtype 5 with C-11ABP688. J Nucl Med 48:247-252. Medline

Andersen P, Bliss TV, Skrede KK (1971) Lamellar organization of hippocampal excitatory pathways. Exp Brain Res 13:222-238. Medline

Banati RB, Newcombe J, Gunn RN, Cagnin A, Turkheimer F, Heppner F, Price G, Wegner F, Giovannoni G, Miller DH, Perkin GD, Smith T, Hewson AK, Bydder G, Kreutzberg GW, Jones T, Cuzner ML, Myers R (2000) The peripheral benzodiazepine binding site in the brain in multiple sclerosis: quantitative in vivo imaging of microglia as a measure of disease activity. Brain 123:2321-2337. CrossRef Medline

Barnes CA, Jung MW, McNaughton BL, Korol DL, Andreasson K, Worley PF (1994) Ltp saturation and spatial-learning disruption: effects of task variables and saturation levels. J Neurosci 14:5793-5806. CrossRef Medline

Bockenstedt LK, Gonzalez DG, Haberman AM, Belperron AA (2012) Spirochete antigens persist near cartilage after murine lyme borreliosis therapy. J Clin Invest 122:2652-2660. CrossRef Medline

Boelen E, Stassen FR, van der Ven AJ, Lemmens MA, Steinbusch HP, Bruggeman CA, Schmitz C, Steinbusch HW (2007) Detection of amyloid beta aggregates in the brain of $\mathrm{BALB} / \mathrm{c}$ mice after chlamydia pneumoniae infection. Acta Neuropathol 114:255-261. CrossRef Medline

Brown JP, Zachary JF, Teuscher C, Weis JJ, Wooten RM (1999) Dual role of interleukin-10 in murine lyme disease: regulation of arthritis severity and host defense. Infect Immun 67:5142-5150. Medline

Buscombe J, Signore A (2003) FDG-PET in infectious and inflammatory disease. Eur J Nucl Med Mol Imaging 30:1571-1573. CrossRef Medline

Carlsson A, Waters N, Holm-Waters S, Tedroff J, Nilsson M, Carlsson ML (2001) Interactions between monoamines, glutamate, and GABA in schizophrenia: new evidence. Annu Rev Pharmacol Toxicol 41:237-260. CrossRef Medline

Choi H, Kim YK, Kang H, Lee H, Im HJ, Hwang DW, Kim EE, Chung JK, Lee DS (2014) Abnormal metabolic connectivity in the pilocarpine-induced epilepsy rat model: a multiscale network analysis based on persistent homology. Neuroimage 99:226-236. CrossRef Medline

Compston A, Coles A (2008) Multiple sclerosis. Lancet 372:1502-1517. CrossRef Medline

Cunningham C, Wilcockson DC, Campion S, Lunnon K, Perry VH (2005) Central and systemic endotoxin challenges exacerbate the local inflammatory response and increase neuronal death during chronic neurodegeneration. J Neurosci 25:9275-9284. CrossRef Medline

Czerniawski J, Guzowski JF (2014) Acute neuroinflammation impairs con- text discrimination memory and disrupts pattern separation processes in hippocampus. J Neurosci 34:12470-12480. CrossRef Medline

Dersch R, Sarnes AA, Maul M, Hottenrott T, Baumgartner A, Rauer S, Stich O (2015) Quality of life, fatigue, depression and cognitive impairment in lyme neuroborreliosis. J Neurol 262:2572-2577. CrossRef Medline

Dorr A, Sled JG, Kabani N (2007) Three-dimensional cerebral vasculature of the CBA mouse brain: a magnetic resonance imaging and micro computed tomography study. Neuroimage 35:1409-1423. CrossRef Medline

Ek M, Engblom D, Saha S, Blomqvist A, Jakobsson PJ, Ericsson-Dahlstrand A (2001) Inflammatory response: pathway across the blood-brain barrier. Nature 410:430-431. CrossRef Medline

Evans ME, Pollack M (1993) Effect of antibiotic class and concentration on the release of lipopolysaccharide from Escherichia coli. J Infect Dis 167: 1336-1343. CrossRef Medline

Feder HM Jr, et al. (2007) Current concepts: a critical appraisal of "chronic lyme disease". N Engl J Med 357:1422-1430. CrossRef Medline

Glass CK, Saijo K, Winner B, Marchetto MC, Gage FH (2010) Mechanisms underlying inflammation in neurodegeneration. Cell 140:918-934. CrossRef Medline

Gunn RN, Lammertsma AA, Hume SP, Cunningham VJ (1997) Parametric imaging of ligand-receptor binding in PET using a simplified reference region model. Neuroimage 6:279-287. CrossRef Medline

Hammers A, Koepp MJ, Richardson MP, Hurlemann R, Brooks DJ, Duncan JS (2003) Grey and white matter flumazenil binding in neocortical epilepsy with normal MRI: a PET study of 44 patients. Brain 126:1300-1318. CrossRef Medline

Hildenbrand P, Craven DE, Jones R, Nemeskal P (2009) Lyme neuroborreliosis: manifestations of a rapidly emerging zoonosis. Am J Neuroradiol 30:1079-1087. CrossRef Medline

Ivenshitz M, Segal M (2010) Neuronal density determines network connectivity and spontaneous activity in cultured hippocampus. J Neurophysiol 104:1052-1060. CrossRef Medline

Kehoe J, Vulfius C (2000) Independence of and interactions between GABA-, glutamate-, and acetylcholine-activated cl conductances in aplysia neurons. J Neurosci 20:8585-8596. CrossRef Medline

Kim KM, Vicenty J, Palmore GT (2013) The potential of apolipoprotein E4 to act as a substrate for primary cultures of hippocampal neurons. Biomaterials 34:2694-2700. CrossRef Medline

Klich MA, Arthur KS, Lax AR, Bland JM (1994) Iturin-a: a potential new fungicide for stored grains. Mycopathologia 127:123-127. CrossRef Medline

Kujala J, Jung J, Bouvard S, Lecaignard F, Lothe A, Bouet R, Ciumas C, Ryvlin P, Jerbi K (2015) Gamma oscillations in V1 are correlated with GABA(A) receptor density: a multi-modal MEG and flumazenil-PET study. Sci Rep 5:16347. CrossRef Medline

Lien E, Sellati TJ, Yoshimura A, Flo TH, Rawadi G, Finberg RW, Carroll JD, Espevik T, Ingalls RR, Radolf JD, Golenbock DT (1999) Toll-like receptor 2 functions as a pattern recognition receptor for diverse bacterial products. J Biol Chem 274:33419-33425. CrossRef Medline

Markram H, Toledo-Rodriguez M, Wang Y, Gupta A, Silberberg G, Wu C (2004) Interneurons of the neocortical inhibitory system. Nat Rev Neurosci 5:793-807. CrossRef Medline

Mora F, Segovia G, Del Arco A (2008) Glutamate-dopamine-GABA interactions in the aging basal ganglia. Brain Res Rev 58:340-353. CrossRef Medline

Nau R, Brück W (2002) Neuronal injury in bacterial meningitis: mechanisms and implications for therapy. Trends Neurosci 25:38-45. CrossRef Medline

Oh SW, et al. (2014) A mesoscale connectome of the mouse brain. Nature 508:207-214. CrossRef Medline

O'Neill LA, Golenbock D, Bowie AG (2013) The history of toll-like receptors: redefining innate immunity. Nat Rev Immunol 13:453-460. CrossRef Medline

Oschmann P, Dorndorf W, Hornig C, Schäfer C, Wellensiek HJ, Pflughaupt KW (1998) Stages and syndromes of neuroborreliosis. J Neurol 245: 262-272. CrossRef Medline

Parente A, Vállez Garcia D, Shoji A, Lopes Alves I, Maas B, Zijlma R, Dierckx RA, Buchpiguel CA, de Vries EF, Doorduin J (2017) Contribution of neuroinflammation to changes in $[(11) \mathrm{C}]$ flumazenil binding in the rat brain: evaluation of the inflamed pons as reference tissue. Nucl Med Biol 49:50-56. CrossRef Medline

Parthasarathy G, Fevrier HB, Philipp MT (2013) Non-viable borrelia burg- 
dorferi induce inflammatory mediators and apoptosis in human oligodendrocytes. Neurosci Lett 556:200-203. CrossRef Medline

Rambukkana A, Zanazzi G, Tapinos N, Salzer JL (2002) Contact-dependent demyelination by mycobacterium leprae in the absence of immune cells. Science 296:927-931. CrossRef Medline

Ramesh G, Alvarez AL, Roberts ED, Dennis VA, Lasater BL, Alvarez X, Philipp MT (2003) Pathogenesis of lyme neuroborreliosis: borrelia burgdorferi lipoproteins induce both proliferation and apoptosis in rhesus monkey astrocytes. Eur J Immunol 33:2539-2550. CrossRef Medline

Reuber M, Mitchell AJ, Howlett SJ, Crimlisk HL, Grünewald RA (2005) Functional symptoms in neurology: questions and answers. J Neurol Neurosurg Psychiatry 76:307-314. CrossRef Medline

Ryvlin P, Bouvard S, Le Bars D, De Lamérie G, Grégoire MC, Kahane P, Froment JC, Mauguière F (1998) Clinical utility of flumazenil-PET versus [18F] fluorodeoxyglucose-PET and MRI in refractory partial epilepsy: a prospective study in 100 patients. Brain 121:2067-2081. CrossRef Medline

Saijo K, Winner B, Carson CT, Collier JG, Boyer L, Rosenfeld MG, Gage FH, Glass CK (2009) A Nurr1/CoREST pathway in microglia and astrocytes protects dopaminergic neurons from inflammation-induced death. Cell 137:47-59. CrossRef Medline

Shiga T, Ikoma K, Katoh C, Isoyama H, Matsuyama T, Kuge Y, Kageyama H, Kohno T, Terae S, Tamaki N (2006) Loss of neuronal integrity: a cause of hypometabolism in patients with traumatic brain injury without MRI abnormality in the chronic stage. Eur J Nucl Med Mol Imaging 33:817822. CrossRef Medline

Singh P, Cameotra SS (2004) Potential applications of microbial surfactants in biomedical sciences. Trends Biotechnol 22:142-146. CrossRef Medline van de Beek D, de Gans J, Spanjaard L, Weisfelt M, Reitsma JB, Vermeulen M (2004) Clinical features and prognostic factors in adults with bacterial meningitis. N Engl J Med 351:1849-1859. CrossRef Medline

Vasek MJ, et al. (2016) A complement-microglial axis drives synapse loss during virus-induced memory impairment. Nature 534:538-543. CrossRef Medline

Vivash L, Gregoire MC, Lau EW, Ware RE, Binns D, Roselt P, Bouilleret V, Myers DE, Cook MJ, Hicks RJ, O’Brien TJ (2013) F-18-flumazenil: a gamma-aminobutyric acid A-specific PET radiotracer for the localization of drug-resistant temporal lobe epilepsy. J Nucl Med 54:1270-1277. CrossRef Medline

Wippel C, Maurer J, Förtsch C, Hupp S, Bohl A, Ma J, Mitchell TJ, Bunkowski S, Brück W, Nau R, Iliev AI (2013) Bacterial cytolysin during meningitis disrupts the regulation of glutamate in the brain, leading to synaptic damage. PloS Pathog 9:e1003380. CrossRef Medline

Xanthos DN, Sandkühler J (2014) Neurogenic neuroinflammation: inflammatory CNS reactions in response to neuronal activity. Nat Rev Neurosci 15:43-53. CrossRef Medline 